\title{
Commitment in First-price Auctions
}

\author{
Yunjian $\mathrm{Xu}^{*}$ and Katrina Ligett ${ }^{\dagger}$
}

November 12, 2014

\begin{abstract}
We study a variation of the single-item sealed-bid first-price auction wherein one bidder (the leader) publicly commits to a strategy before the others submit their bids. We fully characterize the committed mixed strategy that is optimal for the leader, and find that the leader and the follower with the highest valuation strictly benefit from the commitment. We further show that compared with the simultaneous first-price auction, the leader's optimal commitment yields the same net utility benefit to each of the two highest-valued bidders. As a result, these two bidders' incentives are aligned, facilitating coordination and implementation of the commitment.
\end{abstract}

\footnotetext{
${ }^{*}$ Corresponding author. Engineering Systems and Design, Singapore University of Technology and Design; xuyunjian@gmail.com.

${ }^{\dagger}$ Division of the Humanities and Social Sciences, California Institute of Technology; katrina@caltech.edu.

The authors wish to thank Marina Agranov, John Ledyard, Bernhard von Stengel, Adam Wierman, John Wooders, and the participants of Dagstuhl seminar on the Interface of Computation, Game Theory, and Economics at Wadern, Germany, April 2013, and the Social Sciences Brown Bag Seminar at Califnornia Institute of Technology, April 2013, for their helpful comments. This research was supported in part by the MIT-SUTD International Design Center (IDC) Grant IDG21400103, NSF grants CCF-0910940 and CNS-1254169, the Charles Lee Powell Foundation, and a Microsoft Research Faculty Fellowship.
} 


\section{Introduction}

This paper studies the impact of commitment in first-price auctions. We consider the effects on the equilibrium strategies of the players, and on player and auctioneer payoffs, when one player is given the option to publicly pre-commit to a distribution from which her bid will be drawn. For certain players, we demonstrate significant potential benefits of commitment, establishing a clear incentive for the emergence of formal or informal commitment mechanisms.

This work is closely related to the study of collusive bidder behavior in auctions, which has received extensive attention due to the serious threat it poses to real markets $[1,2]$. Commitment as we study it may be seen as a weak form of bidder collusion that nonetheless has a number of appealing properties - no monetary transfers, limited coordination, and significant potential financial gains.

The present paper belongs to the literature that establishes bidders' collusive behavior as a noncooperative game theoretic solution in first- and second-price auctions. Marshall and Marx [3] compare the collusive behavior (induced by a cartel) in first and second auctions. Eso and Schummer [4] show that the second-price auction is not bribe-proof: a simple form of collusion where one bidder may bribe another to leave the auction occurs at every robust sequential equilibrium. Kivetz and Tauman [5] study a simple collusive mechanism wherein the highest valued bidder bribes the second highest valued bidder to bid the auctioneer's reservation price, in a first-price auction setting with complete information that is similar to ours.

While most previous work in this literature focuses on collusive mechanisms involving monetary transfers (among cartel members), this paper analyzes a coordinative mechanism that allows the bidders to collude without monetary transfers. This latter form of collusion, which is referred to as implicit collusion in McAfee and McMillan [6], is harder to detect; as a result, bidders may prefer implicit collusion, especially when the risk of detection of transfers is sufficiently high. 
As show in [6], in a one-shot first-price auction where transfers are not allowed, the best a cartel can do is either to randomly pick the winner or to have its members bid competitively. For repeated auctions, on the other hand, there has been a stream of recent work showing that collusion is possible through bid rotation schemes, even without side payments $[7,8,9]$. This paper attempts to establish a coordinative mechanism (that allows bidders to collude without side payment) as a solution to a Stackelberg game, through fully understanding the effect of commitments in a single-item first-price auction. $^{1}$

We consider a two-stage Stackelberg game, where in the first round one bidder (the leader) is allowed to publicly commit to a mixed strategy; in the second round, the other bidders submit their bids simultaneously. Given the publicly known commitment of the leader, the other bidders simultaneously play (possibly randomized) actions in the second stage. The outcome of the game depends on the realizations of the random variable according to which the leader commits to bid and of the followers' randomized actions: the bidder with the highest realized bid wins the item and pays her bid.

Intuitively, the leader may have incentive to commit to a distribution with support below what she would have played in a simultaneous first price auction, because in doing so, she induces her opponents to lower their bids as well. In this way, the leader may sacrifice winning the auction on occasion in exchange for winning at a better price when she does win. Our results provide support for this intuition. For example, we see that even a very simple form of commitment, in which the leader announces to bid zero (effectively, to exit the auction) with some positive probability $p$ and to bid some other announced real number with probability $1-p$, can strictly (and significantly) benefit both bidders in a simple two-bidder setting (cf. Example 1).

The insight that the commitment power usually confers a strategic advantage for the leader is well known $[10,11]$. In work that partially motivates the present study, von

\footnotetext{
${ }^{1}$ Commitment in a (private value) second-price auction is less interesting, because it remains optimal for the followers to bid their true valuations, regardless of the (possibly mixed) commitment made by the leader. Effectively, the second-price auction breaks the coordination that commitments allow.
} 
Stengel and Zamir [12] show that in a two-player Stackelberg game with finite action space, the leader always benefits from the power to commit to mixed strategies; when there is more than one follower, however, a leader may strictly lose by being required to commit to a mixed strategy. Unlike in [12], the focus of this paper is on deriving a complete characterization of optimal commitments, for a more technically challenging setting with continuous action space. We also show that pre-play commitment in firstprice auctions always strictly benefits the leader even if there is more than one follower, as long as the leader's valuation is among the top two.

Our analysis begins with the case wherein all bidders' valuations are commonly known. ${ }^{2}$ We completely characterize the optimal commitment strategy for the leader in terms of the bidder valuations, for arbitrary numbers of bidders. The characterized optimal commitment, together with best responses on the part of the followers, forms a subgame perfect equilibrium (SPE). We then apply the methodology developed for the complete information setting to a Bayesian setting with two bidders, where each bidder knows her own valuation, and the leader knows only a prior distribution on the follower's valuation.

We find that if the leader has the highest or the second highest valuation, then the two bidders with highest valuations strictly benefit from the presence of a committing bidder. This result establishes the leader's optimal commitment as a coordinative mechanism that allows the top two valued bidders to collude without money transfer. Somewhat surprisingly, compared with the simultaneous first-price auction, the leader's optimal commitment yields both bidders the same net utility benefit. This observation may eliminate possible conflicts (between the top two valued bidders) over possible ways to coordinate (e.g., who should commit and who should follow; cf. the discussion following Theorem 1).

The result derived in this paper is quantitatively similar to those established in

\footnotetext{
${ }^{2}$ We note that the complete information setting is somewhat standard in the literature on commitments in Stackelberg games (see [5, 12]), and often yields a nontrivial optimal commitment for the leader.
} 
Rubinstein and Wolinsky [13] and Vincent [14], that in a multi-stage game, a seller who interacts with only one buyer at a time may give up the good for free at equilibrium, even if the valuations of all buyers are identical and commonly known. This is because the separation of the buyers in bidding stages supports buyers' implicitly collusive behavior at an equilibrium, and confers a strategic disadvantage on the seller. In a repeated first-price auction setting (where the seller interacts with all bidders at every stage), on the other hand, there exists a symmetric SPE at which the seller sells the good at the buyer's valuation at the first stage, even when only two buyers bid simultaneously at every stage [14]. The present work shows that pre-play commitment supports buyers' collusive equilibrium behavior and partially eliminates the competition observed in repeated first-price auctions, in a setting (similar to that of $[13,14]$ ) with commonly known but possibly heterogeneous bidder valuations.

It is worth noting that a leader's optimal commitment may result in inefficient outcomes, i.e., the highest valued bidder does not always win the item. Because the leader's optimal commitment benefits the bidders but hurts the welfare, it must decrease the auctioneer's revenue. Indeed, the auctioneer's revenue strictly decreases whenever the leader (and the highest valued follower) strictly benefits from the commitment. This is the case when the leader is allowed to make arbitrary randomized commitments and she has at least the second highest valuation, or even when the leader can only make the simplest randomized commitments with support only on two bids, and there are two bidders with $v_{1}<2 v_{2}$ (where $v_{1}$ is the leader's valuation, and $v_{2}$ the follower's valuation).

Although implementation of commitment to a randomized bid presents a real practical challenge, there are a number of reasons we argue that it is an interesting object of study. First, because we demonstrate that successful implementation of such a commitment scheme would have significant economic benefits for bidders, there is a clear incentive for bidders to establish a credible commitment scheme, perhaps through a trusted authority (e.g., computer programs [15]) or a reputation system via repeated 
play. ${ }^{3}$ Second, we show in Example 1 that even a very simple form of commitment, in which the leader commits that with a certain probability she will exit the game without bidding, still confers significant benefits. This paper also gives the tools to understand the impact of such simpler, easier to implement, commitment schemes.

The paper is organized as follows. In the next section, we formulate the studied auction model as a Stackelberg game. In Section 3, we characterize the leader's optimal commitment, for both the two-bidder and the multi-bidder cases. We also show that, when the leader has one of the two highest valuations, the leader's optimal commitment yields the two highest-valued bidders expected payoffs higher than those achieved at a Nash equilibrium of the corresponding simultaneous first-price auction. In Section 4, we consider a setting where the leader is restricted to announce a discrete random variable of which the range has a cardinality no more than $t$. We characterize the leader's optimal commitment as a solution to an optimization problem, and show that the leader can approximately achieve her highest expected payoff (that could be achieved by making arbitrary randomized commitments) by committing to bid a discrete random variable that is optimal in her restricted action space, with sufficiently large $t$. In Section 5 , we consider a two-bidder Bayesian setting, and characterize a leader's optimal commitment through the methodology developed in Section 3. Finally, in Section 6, we make some brief concluding remarks.

\section{Formulation}

We study a variation of the standard single-item sealed-bid first-price auction, wherein one bidder (we will refer to her as bidder 1) can publicly make a commitment to play a (possibly mixed) strategy, before the other bidders submit their bids.

For $i=1, \ldots, n$, let $v_{i}>0$ denote bidder $i$ 's valuation for the item. We consider a

\footnotetext{
${ }^{3}$ In a repeated game, if there is positive prior probability that a patient long-run player is committed to play a certain fixed strategy, then at an equilibrium the player approximately gets at least her (static) Stackelberg payoff $[16,17,18]$.
} 
complete information setting where the $n$ bidders' valuations are common knowledge. We formulate the auction as a two-stage game known as a Stackelberg game: in the first stage, bidder 1 announces her bid (to be submitted in the auction) as a random variable, $X_{1}: \Omega \rightarrow[0, \infty)$, defined on a probability space $(\Omega, \mathcal{B}, \mathbb{P})$, where $\Omega=[0,1]$, $\mathcal{B}$ is the Borel $\sigma$-field, and $\mathbb{P}$ is the Lebesgue measure. In order to make bidder 1 's expected payoff well-defined, we restrict bidder 1's action space (at the first stage) to the set of random variables that have an expectation, which is denoted by $\mathcal{X}$ in this paper.

At the second stage, the remaining $n-1$ bidders submit their bids simultaneously. These $n-1$ bidders are allowed to use a randomized strategy. Formally, for $i=2, \ldots, n$, bidder $i$ 's strategy, $X_{i}$, is a mapping from the set of possible commitments, $\mathcal{X}$, to the set of random variables $\mathcal{X}$. For $i=2, \ldots, n$, if for any commitment (made by bidder 1) $X_{1} \in \mathcal{X}$, the range of $X_{i}\left(X_{1}\right)$ is a singleton, then we say that bidder $i$ uses a pure strategy; that is, bidder $i$ 's pure strategy is a mapping from $\mathcal{X}$ to $[0, \infty)$.

For $i=1, \ldots, n$, the bid submitted by bidder $i$ is a realization of the random variable $X_{i}$. The outcome of the game depends on these realizations, and the bidder who bids highest wins the item and pays her bid. Without loss of generality, let bidder 2 have the highest valuation among the $n-1$ bidders excluding bidder 1 .

We use $\pi_{i}\left(X_{1}, \ldots, X_{n}\right)$ to denote bidder $i$ 's expected payoff achieved under a strategy profile $\left(X_{1}, \ldots, X_{n}\right)$. At a Subgame Perfect Equilibrium (SPE), the remaining $n-1$ bidders (excluding bidder 1) play a Nash equilibrium in the subgame that follows bidder 1's commitment, and bidder 1 maximizes her expected payoff given the strategy profile used by the remaining $n-1$ bidders. Formally, a strategy profile $\left(X_{1}, \ldots, X_{n}\right)$ is an SPE of the game, if for $i=2, \ldots, n$,

$$
\pi_{i}\left(X_{i}, \tilde{X}_{1}, \mathbf{X}_{-i}\right) \geq \pi_{i}\left(\widetilde{X}_{i}, \widetilde{X}_{1}, \mathbf{X}_{-i}\right), \quad \forall \tilde{X}_{1} \in \mathcal{X}, \quad \forall \tilde{X}_{i}: \mathcal{X} \rightarrow \mathcal{X}
$$

where $\mathbf{X}_{-i}$ denotes the strategy profile used by the other bidders excluding bidder 1 
and bidder $i$, and

$$
\pi_{1}\left(X_{1}, X_{2}\left(X_{1}\right), \ldots, X_{n}\left(X_{1}\right)\right) \geq \pi_{1}\left(\tilde{X}_{1}, X_{2}\left(\tilde{X}_{1}\right), \ldots, X_{n}\left(\tilde{X}_{1}\right)\right), \quad \forall \tilde{X}_{1} \in \mathcal{X}
$$

\section{Optimal Commitment}

In this section, we characterize bidder 1's optimal commitment that maximizes her expected payoff, conditioned on the other bidders playing a Nash equilibrium of the subgame following bidder 1's commitment. We first analyze optimal commitments in the two bidder case, and then proceed to study a more general setting with $n$ bidders.

\subsection{Two bidder case}

For a two-bidder model, we fully characterize the "optimal leadership strategy", a commitment to play a mixed strategy that maximizes the leader's expected payoff. To characterize the SPE that maximizes the leader's expected payoff, we assume that bidder 2 wins the item if there is a tie. We consider the following pure strategy of bidder 2:

$$
b_{2}^{*}(X)=\min \left\{\operatorname{argmax}_{b \in\left[0, v_{2}\right]}\left\{F_{X}(b)\left(v_{2}-b\right)\right\}\right\}, \quad \forall X \in \mathcal{X}
$$

where $F_{X}(\cdot)$ is the cumulative distribution function $(\mathrm{CDF})$ of the random variable $X$ the leader commits to. We will show in Lemma 1 that the pure strategy defined in (1) is legitimate, and is therefore optimal for bidder 2 .

Note that the best response (of the follower) characterized in (1) is most preferred by the leader. Before proceeding, we emphasize that the tie-breaking assumption and the assumption that bidder 2 always chooses the leader's favorite best response ${ }^{4}$ are

\footnotetext{
${ }^{4}$ Indeed, as noted by Maschler [19] and von Stengel and Zamir [12], if the follower did not choose the best response that is most favorable to the leader, the leader could induce such behavior at an SPE by slightly modifying her commitment.
} 
only used to characterize the SPE that maximizes the leader's expected payoff. Neither assumption plays a major role in our main result: in Theorem 1 we show that this SPE maximizes the leader's expected payoff among all SPEs (of all possible models with different tie-breaking rules), and that bidder 1 can approximately secure this highest expected payoff, regardless of the best response chosen by bidder 2, and regardless of who will win the item at a tie.

The following definition formulates the highest expected payoff that bidder 1 can "secure", regardless of which best response the followers choose.

Definition 1. In an $n$-bidder model for $n \geq 2$, we say bidder 1 can secure an expected payoff $s \geq 0$, if there exists an commitment $X_{1}$ such that in the subgame (among the other $n-1$ bidders) that follows commitment $X_{1}$, there exists at least one Nash equilibrium, and every Nash equilibrium of the subgame yields bidder 1 an expected payoff no less than s. Let $\mathcal{S}$ denote the set of expected payoffs bidder 1 can secure. We define $\bar{\pi}_{1}=\sup _{s}\{s \in \mathcal{S}\}$, which is the supremum of the expected payoffs bidder 1 can achieve by way of any commitment.

Definition 1 is for a general $n$-bidder model, and will be used throughout the entire paper. We note that $\bar{\pi}_{1}$ is closely related to the concept of "Stackelberg payoff", which is defined as the leader's maximum payoff when the followers choose the Nash equilibrium that is worst for the leader [20]. It is straightforward to see that the Stackelberg payoff belongs to the set $\mathcal{S}$, and therefore is no more than $\bar{\pi}_{1}$.

Lemma 1. Suppose that bidder 2 wins the item if there is a tie. For any random variable $X_{1}$ announced by player 1 , the strategy characterized in (1) is legitimate: that is, the set of maximizers is nonempty, and there exists a minimum of the set.

Proof. We first argue that the supremum of bidder 2's expected payoff is attained within the interval $\left[0, v_{2}\right]$. Note that for any commitment $X_{1}$, its $\operatorname{CDF} F_{X_{1}}(b)$ is rightcontinuous and has at most countably many discontinuities; thus, so does bidder 2's 
expected payoff $\pi_{2}\left(X_{1}, b\right)$. At any discontinuity $b$ of $F_{X_{1}}$, we have

$$
\limsup _{x \rightarrow b^{-}} \pi_{2}\left(X_{1}, x\right)=\limsup _{x \rightarrow b^{-}} F_{X_{1}}(x)\left(v_{2}-x\right) \leq F_{X_{1}}(b)\left(v_{2}-b\right)=\pi_{2}\left(X_{1}, b\right)
$$

where the inequality holds because $F_{X_{1}}$ is nondecreasing. Because $\pi_{2}\left(X_{1}, b\right)$ is rightcontinuous in $b$, it follows that $\pi_{2}\left(X_{1}, b\right)$ is upper semi-continuous. It must have a maximum on the compact set $\left[0, v_{2}\right]$.

It remains to show that the set of maximizers has a minimum. If the set of maximizers is finite, then we are done. Suppose now the set is infinite. Let $\underline{b}$ denote the infimum of the set of maximizers. There exists a sequence of maximizers, $\left\{b^{k}\right\}$, which is decreasing and converges to the infimum $\underline{b}$. Then $\underline{b}$ also maximizes bidder 2's expected payoff, i.e.,

$$
\pi_{2}\left(X_{1}, \underline{b}\right)=\lim _{k \rightarrow \infty} \pi_{2}\left(X_{1}, b^{k}\right)=\max _{b \in\left[0, v_{2}\right]}\left\{F_{X_{1}}(b)\left(v_{2}-b\right)\right\}
$$

where the first equality holds because $\pi_{2}\left(X_{1}, b\right)$ is right-continuous.

We are now ready to fully characterize the leader's optimal commitment in the following theorem. According to this commitment, the leader leaves the auction (submits a zero bid) with a certain positive probability. The commitment makes bidder 2 (the follower) indifferent among a compact set of actions. Along the equilibrium path of the SPE, bidder 2 chooses the best response that is most favorable to the leader, i.e., to bid zero. We will also show that the (positive) net benefit of collusion is equally distributed among the two bidders.

Theorem 1. For the two-bidder Stackelberg game considered in this paper we have the following.

1. Suppose that bidder 2 wins the item if there is a tie. A commitment $\bar{X}_{1}$ with the 
following $C D F$

$$
F_{\bar{X}_{1}}(x)= \begin{cases}0, & \text { if } x<0, \\ \frac{v_{2} e^{-v_{1} / v_{2}}}{v_{2}-x}, & \text { if } 0 \leq x \leq v_{2}\left(1-e^{-v_{1} / v_{2}}\right), \\ 1, & \text { if } x>v_{2}\left(1-e^{-v_{1} / v_{2}}\right),\end{cases}
$$

together with bidder 2's strategy defined in Eq. (1), form an SPE of the game. Bidder 2 bids zero on the equilibrium path of the SPE, i.e., $b_{2}^{*}\left(\bar{X}_{1}\right)=0$.

2. At the above SPE, bidder 1 obtains an expected payoff of $v_{1}-v_{2}\left(1-e^{-v_{1} / v_{2}}\right)$, and bidder 2's expected payoff is $v_{2} e^{-v_{1} / v_{2}}$. This SPE yields each bidder an expected payoff strictly higher than any Nash equilibrium of the corresponding simultaneous complete-information first-price auction, and further, the positive net benefit is equally distributed among the two bidders.

3. The above SPE yields bidder 1 the highest expected payoff among all SPEs (of all possible models with different tie-breaking rules).

4. Bidder 1 can approximately secure this highest expected payoff, regardless of the tie-breaking rule and the best response chosen by bidder 2 . That is, bidder 1's expected payoff achieved at the SPE equals $\bar{\pi}_{1}$ (cf. Definition 1). ${ }^{5}$

Note that the strategy $b_{2}^{*}$ defined in (1) is a best response of bidder 2 to any commitment $X$. To argue that $\left(\bar{X}_{1}, b_{2}^{*}\right)$ is an SPE, we therefore only need to show that the commitment $\bar{X}_{1}$ maximizes $\pi_{1}\left(X_{1}, b_{2}^{*}\right)$ among all possible commitments $X_{1}$ (cf. Example 97.3 of Osborne and Rubinstein [21]). The second part of the theorem shows that both bidders must strictly benefit from the commitment characterized in the first part.

Somewhat surprisingly, compared with a Nash equilibrium of the simultaneous complete-information first-price auction, the SPE characterized in Part 1 yields both

\footnotetext{
${ }^{5}$ We will show in the proof of this theorem that the value of $\bar{\pi}_{1}$ does not depend on the tie-breaking rule.
} 
bidders the same net utility benefit, i.e.,

$$
\pi_{1}\left(X_{1}, b_{2}^{*}\right)-\max \left\{0, v_{1}-v_{2}\right\}=\pi_{2}\left(X_{1}, b_{2}^{*}\right)-\max \left\{0, v_{2}-v_{1}\right\}>0
$$

where $\max \left\{0, v_{i}-v_{j}\right\}$ is bidder $i$ 's maximum payoff that could be achieved at a Nash equilibrium of the corresponding simultaneous complete-information first-price auction (cf. the discussion following Lemma 3). ${ }^{6}$ We note that a similar argument is true for the multi-bidder case considered in Section 3.2 (cf. Part 2 of Theorem 2).

The surprising fact expressed in Eq. (3) implies a Pareto ordering on the two possible ways to collude (bidder 1 commits vs. bidder 2 commits), and therefore eliminates possible conflicts (between the two bidders) on who should be the leader. To see this, let $\pi_{i}$ denote the expected payoff of bidder $i$ (at the SPE characterized in Theorem 1) when bidder 1 commits, and let $\varphi_{i}$ denote the expected payoff of bidder $i$ (at the SPE that maximizes the leader's expected payoff) when bidder 2 commits. It follows from Eq. (3) that

$$
\pi_{1}-\pi_{2}=v_{1}-v_{2}=\varphi_{1}-\varphi_{2}
$$

So, for example, if bidder 1 prefers to be the leader, then $\pi_{1} \geq \varphi_{1}$, or equivalently, $\pi_{2}+v_{1}-v_{2} \geq \varphi_{2}+v_{1}-v_{2}$, i.e., bidder 2 also prefers the case where bidder 1 is the leader.

Part 3 states that bidder 1 cannot obtain an expected payoff that is higher than $v_{1}-v_{2}\left(1-e^{-v_{1} / v_{2}}\right)$ at an SPE, because at the SPE bidder 2 chooses her best reply that is most favorable to bidder 1 (cf. Eq. (1)). Further, even under a "pessimistic" assumption that bidder 2 chooses the "worst" best response for bidder 1, Part 4 shows that bidder 1 can approximately achieve this expected payoff by adjusting her commitment a slightly (in a way that will be rigorously described at the end of the following proof) to make bidding zero a unique best response of bidder 2 .

\footnotetext{
${ }^{6}$ If $v_{i}>v_{j}$ and bidder $i$ wins the item at a tie, then $\left(v_{j}, v_{j}\right)$ is a pure Nash equilibrium of the simultaneous complete-information first-price auction, which yields bidder $i$ a payoff of $v_{i}-v_{j}$.
} 


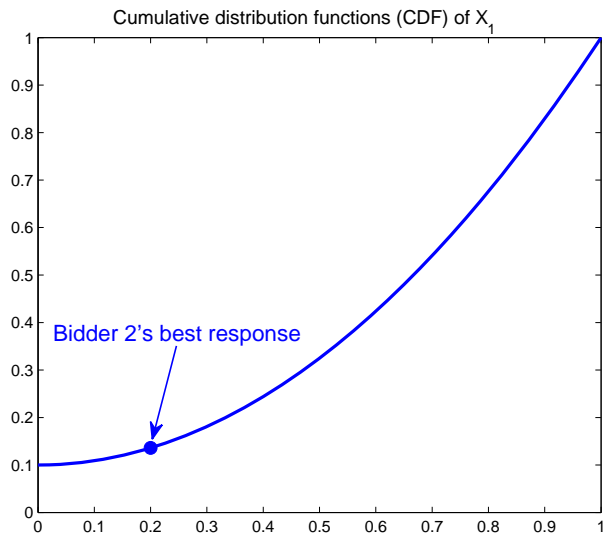

Figure 1: The CDF of the original commitment $X_{1}$.

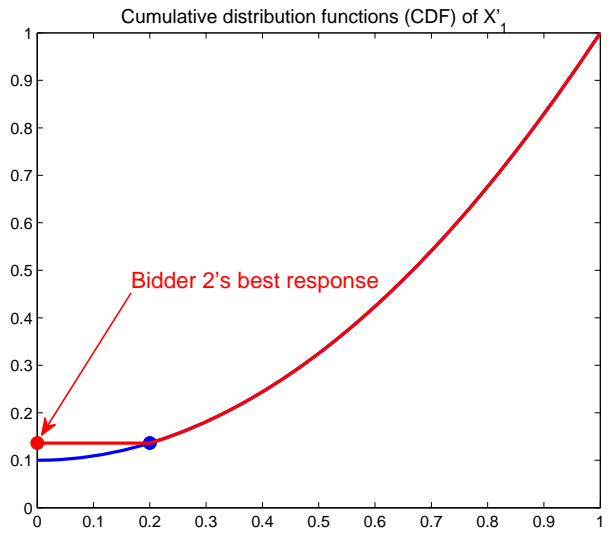

Figure 2: The red curve illustrates the CDF of the modified commitment $X_{1}^{\prime}$.

Proof. To prove that $\left(\bar{X}_{1}, b_{2}^{*}\right)$ is an SPE, we will show that $\bar{X}_{1}$ maximizes $\pi_{1}\left(X_{1}, b_{2}^{*}\right)$ among all possible announcements $X_{1}$, in the following three steps.

Step 1: There exists an optimal commitment under which bidding zero is a best response of bidder 2.

Suppose that bidder 1 commits to submit a random variable $X_{1}$, and suppose by way of contradiction that bidder 2's best response is positive, i.e., $b_{2}^{*}\left(X_{1}\right)>0$. Given the CDF of the random variable $X_{1}$, we define an alternative random variable $X_{1}^{\prime}$ by

$$
F_{X_{1}^{\prime}}(x)= \begin{cases}0, & \text { if } x<0 \\ F_{X_{1}}\left(b_{2}^{*}\left(X_{1}\right)\right), & \text { if } 0 \leq x \leq b_{2}^{*}\left(X_{1}\right), \\ F_{X_{1}}(x), & \text { if } x>b_{2}^{*}\left(X_{1}\right) .\end{cases}
$$

That is, bidder 1 is committed to bid zero with probability $F_{X_{1}}\left(b_{2}^{*}\left(X_{1}\right)\right)$ in this alternative announcement (see Figures 1 and 2 for an illustration of $F_{X_{1}}$ and $F_{X_{1}^{\prime}}$ ). It is not hard to see that under the alternative commitment $X_{1}^{\prime}$, bidder 2's best response 


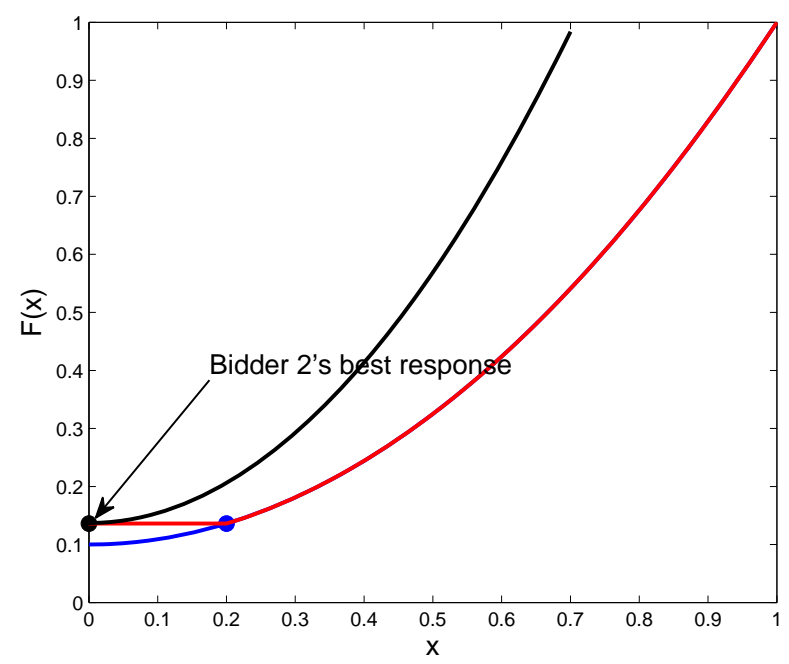

Figure 3: The black curve illustrates the CDF of a commitment $\bar{X}_{1}$ that satisfies condition (5).

is to bid zero, i.e., $b_{2}^{*}\left(X_{1}^{\prime}\right)=0$. It follows that bidder 1 wins the item with an equal probability by committing to bid $X_{1}^{\prime}$. Because bidder 1's expected payment does not rise under this alternative announcement, ${ }^{7}$ it follows that bidder 1 's expected payoff cannot decrease under $X_{1}^{\prime}$, i.e., $\pi_{1}\left(X_{1}^{\prime}, b_{2}^{*}\right) \geq \pi_{1}\left(X_{1}, b_{2}^{*}\right)$.

\section{Step 2: Optimality conditions.}

In this step, we prove that there exists an optimal commitment for bidder 1 whose CDF satisfies

$$
F_{\bar{X}_{1}}(x)= \begin{cases}0, & \text { if } x<0 \\ \frac{v_{2} F_{\bar{X}_{1}}(0)}{v_{2}-x}, & \text { if } 0 \leq x<v_{2}\left(1-F_{\bar{X}_{1}}(0)\right), \\ 1, & \text { if } x \geq v_{2}\left(1-F_{\bar{X}_{1}}(0)\right) .\end{cases}
$$

\footnotetext{
${ }^{7}$ Note that we have assumed that the expectation of $X_{1}$ exists, and thus $X_{1}:[0,1] \rightarrow[0, \infty)$ is Lebesgue integrable. It is easy to check that $X_{1}^{\prime}$ defined in (4) is also Lebesgue integrable, and therefore its expectation exists, i.e., $X_{1}^{\prime} \in \mathcal{X}$.
} 
In Eq. (5), we essentially define a set of random variables parameterized by the probability mass they assign to zero, $F_{\bar{X}_{1}}(0)$.

In Step 1, we have shown that there exists an optimal leader commitment under which bidder 2's best response set includes zero. Let $X_{1}$ be such an optimal commitment; we must have

$$
v_{2} F_{X_{1}}(0) \geq\left(v_{2}-x\right) F_{X_{1}}(x), \quad \forall x \geq 0,
$$

where the left hand side is bidder 2's expected payoff when bidding zero, and the right hand side is her expected payoff when bidding $x$. For a random variable $X_{1}$ that satisfies the condition in (6), if the inequality strictly holds for some $x \in\left[0, v_{2}\left(1-F_{X_{1}}(0)\right)\right]$, i.e., if

$$
F_{X_{1}}(x)<\frac{v_{2} F_{X_{1}}(0)}{v_{2}-x} \leq 1
$$

then we must have $\pi_{1}\left(X_{1}\right) \leq \pi_{1}\left(\bar{X}_{1}\right)$, where $\bar{X}_{1}$ is a random variable satisfying conditions (5). To see this, note that under the commitment $\bar{X}_{1}$, bidder 2's best response remains zero ${ }^{8}$, bidder 1 wins the item with same probability (because $F_{\bar{X}_{1}}(0)=F_{X_{1}}(0)$ ), and bidder 1's expected payment cannot increase under the announcement $\bar{X}_{1}$. It follows that there always exists an optimal announcement (under which it is optimal for bidder 2 to bid zero) that satisfies conditions (5). An illustration is provided in Figure 3, where the red curve representing the CDF of $X_{1}$, and the black curve illustrating the modified announcement $\bar{X}_{1}$ that satisfies the condition in (5).

Step 3: The probability that bidder 1 should leave the auction, $F_{\bar{X}_{1}}(0)$, equals $e^{-v_{1} / v_{2}}$.

\footnotetext{
${ }^{8}$ Actually, the commitment $\bar{X}_{1}$ makes bidder 2 indifferent from any bid in the interval $\left[0, v_{2}\left(1-F_{\bar{X}_{1}}(0)\right)\right]$. Note also that we have assumed bidder 2 would like to choose the smallest component of her best response set.
} 
For the random variable $\bar{X}_{1}$ that satisfies conditions (5), we first argue that its expectation can be calculated as

$$
\mathbb{E}\left\{\bar{X}_{1}\right\}=\int_{0}^{v_{2}\left(1-F_{\bar{X}_{1}}(0)\right)}\left(1-F_{\bar{X}_{1}}(x)\right) d x .
$$

Note that the preceding equality holds for any nonnegative continuous random variable. We will show that it is also valid for a random variable $\bar{X}_{1}$ that satisfies conditions (5), although $\bar{X}_{1}$ is not a continuous random variable.

For notational convenience, we let $\alpha=v_{2}\left(1-F_{\bar{X}}(0)\right)$. From the definition of the random variable $\bar{X}$ in (5), we note that the derivative of its $\mathrm{CDF}, f_{\bar{X}_{1}}$, exists over the domain $(0, \alpha)$. Because the range of $\bar{X}_{1}$ is $[0, \alpha]$, we have

$$
\begin{aligned}
\mathbb{E}\left\{\bar{X}_{1}\right\} & =\lim _{\epsilon \rightarrow 0} \int_{\epsilon}^{\alpha-\epsilon} x f_{\bar{X}_{1}}(x) d x \\
& =\lim _{\epsilon \rightarrow 0} \int_{\epsilon}^{\alpha-\epsilon} \int_{0}^{x} f_{\bar{X}_{1}}(x) d t d x \\
& =\lim _{\epsilon \rightarrow 0} \epsilon \int_{\epsilon}^{\alpha-\epsilon} \mathbb{P}\left(\bar{X}_{1} \in[t, \alpha-\epsilon]\right) d t, \\
& =\lim _{\epsilon \rightarrow 0} \int_{\epsilon}^{\alpha-\epsilon} \mathbb{P}\left(\bar{X}_{1} \in[t, \alpha-\epsilon]\right) d t, \\
& =\lim _{\epsilon \rightarrow 0} \int_{\epsilon}^{\alpha-\epsilon} F_{\bar{X}_{1}}(\alpha-\epsilon)-F_{\bar{X}_{1}}(t) d t, \\
& =\int_{0}^{\alpha} 1-F_{\bar{X}_{1}}(t) d t,
\end{aligned}
$$

where the last equality holds because $F_{\bar{X}_{1}}$ is right-continuous at 0 , and is left continuous at $\alpha$. We now write bidder 1's expected payoff achieved by committing to bid $\bar{X}_{1}$ as a function of $\beta \triangleq F_{\bar{X}_{1}}(0)$ :

$$
\begin{aligned}
\pi_{1}\left(\bar{X}_{1}, b_{2}^{*}\right) & =v_{1}(1-\beta)-\int_{0}^{v_{2}(1-\beta)}\left(1-F_{\bar{X}_{1}}(x)\right) d x \\
& =v_{1}(1-\beta)-v_{2}(1-\beta)+\int_{0}^{v_{2}(1-\beta)} \frac{v_{2} \beta}{v_{2}-x} d x \\
& =\left(v_{1}-v_{2}\right)(1-\beta)-v_{2} \beta \log \beta,
\end{aligned}
$$


where the second equality follows from (5). The derivative of $\pi_{1}\left(\bar{X}_{1}, b_{2}^{*}\right)$ with respect to $\beta$ is $-v_{1}-v_{2} \log \beta$. It follows that bidder 1's expected payoff is (strictly) maximized at $F_{\bar{X}_{1}}(0)=e^{-v_{1} / v_{2}}$. We have shown the random variable in (2) maximizes bidder 1's expected payoff, $\pi_{1}\left(X_{1}, b_{2}^{*}\right)$, among all possible announcements $X$. It follows that $\left(\bar{X}_{1}, b_{2}^{*}\right)$ is an SPE.

Note that we have shown that along the equilibrium path of the SPE the leader obtains an expected payoff of $\pi_{1}\left(\bar{X}_{1}, b_{2}^{*}\right)=v_{1}-v_{2}\left(1-e^{-v_{1} / v_{2}}\right)$. We now show that this expected payoff is higher than that achieved in a simultaneous complete-information first-price auction. It is well known that at any Nash equilibrium of a simultaneous complete-information sealed-bid first-price auction, the bidder who has the highest valuation will bid at least the second highest valuation (see also Lemma 3). Therefore, a Nash equilibrium of the corresponding simultaneous first-price auction (where all bidders have the same valuations as in our Stackelberg model) cannot yield bidder 1 an expected payoff higher than $\max \left\{0, v_{1}-v_{2}\right\}$. We argue that

$$
v_{1}-v_{2}\left(1-e^{-v_{1} / v_{2}}\right)>\max \left\{0, v_{1}-v_{2}\right\}
$$

It is easy to see that $v_{1}-v_{2}\left(1-e^{-v_{1} / v_{2}}\right)>v_{1}-v_{2}$, and therefore the inequality (8) follows from the fact that

$$
v_{2}\left(1-e^{-v_{1} / v_{2}}\right)<v_{1}, \quad v_{1}>0
$$

i.e., the upper endpoint of the range of $\bar{X}$ is less than $v_{1}$. At the SPE, bidder 2's expected payoff is $v_{2} e^{-v_{1} / v_{2}}>0$. From (9) we also have $\pi_{2}\left(\bar{X}_{1}, b_{2}^{*}\right)=v_{2} e^{-v_{1} / v_{2}}>v_{2}-v_{1}$. It follows that $\pi_{2}\left(\bar{X}_{1}, b_{2}^{*}\right)>\max \left\{0, v_{2}-v_{1}\right\}$.

We now argue that the SPE yields bidder 1 the highest expected payoff among all possible SPEs. Let $\left(\hat{X}_{1}, \hat{X}_{2}\right)$ be an SPE of a model with some arbitrary tie-breaking rule $\gamma$, and let $\pi_{1}^{\gamma}\left(\hat{X}_{1}, b_{2}^{*}\right)$ denote bidder 1's expected payoff achieved under the strategy 
profile $\left(\hat{X}_{1}, b_{2}^{*}\right)$ and the tie-breaking rule $\gamma$. We will first show

$$
\pi_{1}\left(\bar{X}_{1}, b_{2}^{*}\right) \geq \pi_{1}^{\gamma}\left(\hat{X}_{1}, b_{2}^{*}\right)
$$

where the left hand side is bidder 1's expected payoff achieved under tie-breaking rule used in Part 1, according to which bidder 2 wins at a tie. If $\gamma$ assigns the item to bidder 2 at a tie, then the inequality in (10) follows from the definition of an SPE. Otherwise, because bidder 2 may lose the item at a tie, she should not bid any real number to which the commitment $\hat{X}_{1}$ puts positive probability mass, i.e., $F_{\hat{X}_{1}}$ must be continuous at $b_{2}^{*}\left(\hat{X}_{1}\right)$. The inequality in (10) then follows from the fact that

$$
\pi_{1}^{\gamma}\left(\hat{X}_{1}, b_{2}^{*}\right)=\pi_{1}\left(\hat{X}_{1}, b_{2}^{*}\right) \leq \pi_{1}\left(\bar{X}_{1}, b_{2}^{*}\right)
$$

i.e., bidder 1 achieve the same expected payoff under the two different tie-breaking rules, $\gamma$ and the one used in Part 1. It follows from the definition of the strategy $b_{2}^{*}$ (cf. (1)) that $\mathbb{P}\left(\hat{X}_{2}\left(\hat{X}_{1}\right) \geq b_{2}^{*}\left(\hat{X}_{1}\right)\right)=1$, and therefore

$$
\pi_{1}\left(\bar{X}_{1}, b_{2}^{*}\right) \geq \pi_{1}^{\gamma}\left(\hat{X}_{1}, b_{2}^{*}\right) \geq \pi_{1}^{\gamma}\left(\hat{X}_{1}, \hat{X}_{2}\right)
$$

where the second inequality is true because bidder 1's expected payoff is non-increasing in bidder 2's action.

Because the preceding argument (together with the inequality in (11)) holds for any announcement $\hat{X}_{1} \in \mathcal{X}$ and any (possibly mixed) Nash equilibrium $\hat{X}_{2}$ of the subgame that follows $\hat{X}_{1}$, it follows that bidder 1 cannot secure a higher expected payoff, i.e., $\pi_{1}\left(\bar{X}_{1}, b_{2}^{*}\right) \geq \bar{\pi}_{1}$. To see that $\pi_{1}\left(\bar{X}_{1}, b_{2}^{*}\right)=\bar{\pi}_{1}$, consider a sequence of announcements $\left\{\bar{X}_{1}^{k}\right\}$

$$
\bar{X}_{1}^{k}(\omega)=\left\{\begin{array}{lll}
\bar{X}_{1}(\omega), & \text { if } \quad & \bar{X}_{1}(\omega)=0 \\
\bar{X}_{1}(\omega)+\frac{1}{k}, & \text { if } & \bar{X}_{1}(\omega)>0
\end{array}\right.
$$


under which bidder 2's unique best response is to bid zero. It is straightforward to check that $\pi_{1}\left(\bar{X}_{1}, b_{2}^{*}\right)=\lim _{k \rightarrow \infty} \bar{\pi}_{1}\left(\bar{X}_{1}^{k}, b_{2}^{*}\right) \leq \bar{\pi}_{1}$.

\subsection{Multi-bidder case}

In this subsection, we study a general $n$-bidder case, wherein bidder 1 makes a commitment and then the remaining $n-1$ bidders (excluding bidder 1 ) act simultaneously. For the $n$-bidder Stackelberg action model considered this paper, we show that at any (possibly mixed) Nash equilibrium of the subgame that follows bidder 1's commitment, the bidder who has the second highest valuation among the remaining $n-1$ bidders (say, bidder 3) cannot obtain a positive expected payoff. This observation implies that in a multi-bidder game, if bidder 1's valuation is less than the second highest, she cannot receive a positive expected payoff at any SPE. On the other hand, for a case where bidder 1 has either the highest or the second highest valuation, we extend the results derived for a two-bidder setting (in Theorem 1) to the $n$-bidder case. Proofs of results derived in this section are given in Appendix A.

Lemma 2. Suppose that bidder 3's valuation is less than bidder 2's, i.e., $v_{3}<v_{2}$. In the subgame that follows any commitment $X_{1}$, bidder 3 obtains zero payoff at any Nash equilibrium.

Note that the result in Lemma 2 is obvious if we restrict our attention to pure Nash equilibria of a subgame. Lemma 2 shows that the result holds at every (possibly mixed) Nash equilibrium.

Lemma 3. Suppose that bidder 3 has the second highest valuation among the $n-1$ bidders (excluding bidder 1), and her valuation is less than bidder 2's valuation, i.e., $v_{3}<v_{2}$. If bidder 2 uses a strategy $X_{2}: \mathcal{X} \rightarrow \mathcal{X}$ at an $S P E$, then $\mathbb{P}\left(X_{2}\left(X_{1}\right) \geq v_{3}\right)=1$ for any $X_{1}$ such that $\mathbb{P}\left(X_{1}<v_{2}\right)>0$.

We emphasize here that both Lemmas 2 and 3, which will serve as a basis for the 
main result of this section (Theorem 2), hold under any tie-breaking rules. We also note that Lemma 3 implies that at any Nash equilibrium of a simultaneous completeinformation sealed-bid first-price auction, the bidder with the highest valuation will bid at least the second highest valuation. Lemma 3 directly leads to the following proposition.

Proposition 1. Suppose that bidder 3 has the second highest valuation among the $n$ bidders, and $v_{3}<v_{2}$. If $v_{1} \leq v_{3}$, then bidder 1 cannot obtain a positive expected payoff at an SPE.

Proof. If bidder 1 commits to some $X_{1}$ such that $\mathbb{P}\left(X_{1}<v_{2}\right)=0$, then she cannot obtain a positive payoff because $v_{1}<v_{2}$. On the other hand, if $\mathbb{P}\left(X_{1}<v_{2}\right)>0$, then Lemma 3 shows that bidder 2's bid is at least $v_{3}$, which is no less than $v_{1}$. It follows that bidder 1 cannot obtain a positive payoff.

Before introducing the main result of this section, we define a pure strategy profile of the $n-1$ bidders:

$$
\overline{\mathbf{b}}=\left(\bar{b}_{2}, \bar{b}_{3}, \ldots, \bar{b}_{n}\right)
$$

where $\bar{b}_{2}$ is defined by

$$
\bar{b}_{2}(X)=\min \left\{\underset{b \in\left[v_{3}, v_{2}\right]}{\operatorname{argmax}}\left\{F_{X}(b)\left(v_{2}-b\right)\right\}\right\}, \quad \forall X \in \mathcal{X},
$$

and $\bar{b}_{i}(X)=v_{i}$, for any $i \geq 3$ and $X \in \mathcal{X}$. Through a similar approach to that used in the proof of Lemma 1, it can be shown that the strategy in (13) is well defined.

Theorem 2. Suppose that bidder 1 has the highest or the second highest valuation, bidder 2's valuation $v_{2}$ is the highest among the remaining $n-1$ bidders, and that bidder 3 has the second highest valuation among the $n-1$ bidders with $v_{3}<v_{2}$. We have the following. 
1. Suppose that bidder 2 wins the item whenever her bid is no less than any other bidder's. Let $C=\left(v_{2}-v_{3}\right) e^{\left(v_{3}-v_{1}\right) /\left(v_{2}-v_{3}\right)}$. A commitment $\bar{X}_{1}$ with the following $C D F$,

$$
F_{\bar{X}_{1}}(x)= \begin{cases}0, & \text { if } x<0, \\ e^{\left(v_{3}-v_{1}\right) /\left(v_{2}-v_{3}\right)}, & \text { if } 0 \leq x \leq v_{3}, \\ \frac{C}{v_{2}-x}, & \text { if } v_{3}<x \leq v_{2}-C, \\ 1, & \text { if } x>v_{2}-C,\end{cases}
$$

together with the strategy profile defined in Eq. (12), form an SPE of the game. Bidder 2 bids $v_{3}$ at the SPE, i.e., $\bar{b}_{2}\left(\bar{X}_{1}\right)=v_{3}$.

2. At the SPE, bidder 1 obtains an expected payoff of $v_{1}-v_{2}+C$, and bidder 2's expected payoff is $C$. The SPE yields both bidder 1 and 2 higher expected payoffs than any Nash equilibrium of the corresponding simultaneous completeinformation first-price auction.

3. The SPE yields bidder 1 the highest expected payoff among all SPEs (of all possible models with different tie-breaking rules).

4. Bidder 1 can approximately secure this highest expected payoff, regardless of the tie-breaking rule. That is, the SPE yields bidder 1 an expected payoff equal to $\bar{\pi}_{1}$ (cf. Definition 1).

At an SPE, Lemma 3 shows that bidder 2 will bid at least $v_{3}$. Because the SPE $\left(\bar{X}_{1}, \overline{\mathbf{b}}\right)$ maximizes bidder 1's expected payoff conditioned on bidder 2's best response $v_{3}$, the SPE yields bidder 1 the highest expected payoff among all SPEs (part 3). Further, bidder 1 can approximately secure this expected payoff by making bidding $v_{3}$ a strict (and unique) best response of bidder 2. Similar to Theorem 1, Parts 3 and 4 hold regardless of the tie-breaking rule. 


\section{Discrete Mixed Strategy}

An interesting variant of our model arises when bidder 1's commitment is restricted to be a discrete random variable, of which the range has a cardinality no more than some positive integer $t$. Compared to the original model where the leader is allowed to announce any arbitrary randomized bids, a commitment to bid a discrete random variable may be more implementable, especially when $t$ is small.

We first study the two-bidder case. In Theorem 3, we characterize the optimal discrete random variable that maximizes bidder 1's expected payoff as an a solution to an optimization problem. The optimal commitment always assigns a positive probability mass to bidding zero. Note that within the restricted action space, bidder 1 cannot obtain her highest expected payoff (actually, bidder 1's expected payoff is maximized by committing to bid a random variable that is neither discrete nor continuous). However, we show in Theorem 4 that bidder 1 can approximately achieve her highest expected payoff (that could be achieved by committing to arbitrary randomized bids) by committing to bid a discrete random variable that is optimal in her restricted action space, with sufficiently large $t$. We then extend these results to a multi-bidder setting. All proofs in this section are deferred to Appendix B.

\subsection{Two-bidder case}

Theorem 3. Suppose that bidder 1's action space (at the first stage) is restricted to the set of discrete random variables whose ranges have a cardinality no more than $t$, for some fixed integer $t \geq 2$. We have the following.

1. Suppose that bidder 2 wins the item if there is a tie ${ }^{9}$. A commitment $\left\{\left(a_{i}^{*}, p_{i}^{*}\right)\right\}_{i=1}^{t}$ (to bid $a_{i}^{*}$ with probability $p_{i}^{*}$ ), which is an optimal solution to the following opti-

\footnotetext{
${ }^{9}$ Note that this assumption is needed only to characterize the SPE (cf. the discussion following Definition $1)$.
} 
mization problem,

$$
\begin{aligned}
& \text { maximize } \quad v_{1}\left(1-p_{1}\right)-\sum_{i=1}^{t} a_{i} p_{i} \\
& \text { subject to } \quad \sum_{i=1}^{t} p_{i}=1 \text {, } \\
& p_{i} \geq 0, \quad i=1, \ldots, t, \\
& a_{1}=0, \quad a_{i}=v_{2}-\frac{v_{2} p_{1}}{\sum_{k=1}^{i} p_{k}}, \quad i=2, \ldots, t
\end{aligned}
$$

together with bidder 2's strategy defined in Eq. (1), form an SPE of the game. Bidder 2 bids zero at the SPE, and obtains an expected payoff of $v_{2} p_{1}^{*}$.

2. The SPE yields bidder 1 the highest expected payoff among all SPEs (of all possible models with different tie-breaking rules).

We note that the optimization problem in (15) essentially has $t-1$ variables: given $p_{1}, \ldots, p_{t-1}$, the other parameters, $p_{t}, a_{2}, \ldots, a_{t}$, are determined by the equality constraints of Problem (15). Under bidder 1's commitment $\left\{\left(a_{i}, p_{i}\right)\right\}_{i=1}^{t}$, because bidder 2 wins the item at a tie, it follows that bidder 2's best response must belong to the set $\left\{a_{1}, \ldots, a_{t}\right\}$. Similar to the unrestricted case studied in Section 3.1, bidder 1's expected payoff is maximized when bidder 2 is indifferent among these choices, i.e.,

$$
v_{2} p_{1}=\left(v_{2}-a_{i}\right) \sum_{k=1}^{i} p_{k}, \quad i=2, \ldots, t
$$

which implies the third constraint of Problem (15). We note that even if bidder 2 chooses a best response different from that characterized in Eq. (1), bidder 1 can approximately achieve this highest expected payoff by adjusting her announcement infinitesimally, to make bidding zero a unique best response for bidder 2 .

In the following example, we consider an interesting special case with $t=2$. Implementation of such a commitment should be much more feasible, compared with the implementation of commitments to arbitrary mixed strategies. 
Example 1. Suppose that bidder 1 makes an announcement with $t=2$. From Theorem 3 we have $a_{1}^{*}=0$, and

$$
p_{1}^{*}=1-\frac{a_{2}^{*}}{v_{2}}
$$

Note that because $p_{1}^{*} \geq 0$, we have $a_{2}^{*} \leq v_{2}$. Bidder 1's expected payoff is given by

$$
\left(v_{1}-a_{2}^{*}\right)\left(1-p_{1}^{*}\right)=\left(v_{1}-a_{2}^{*}\right) \frac{a_{2}^{*}}{v_{2}}
$$

which is maximized at $a_{2}^{*}=\min \left\{v_{1} / 2, v_{2}\right\}$. If $v_{1} \geq 2 v_{2}$, then bidder 1 should announce to bid $v_{2}$ with probability 1 , as she would do in a simultaneous complete-information sealed-bid first-price action.

When $v_{1}<2 v_{2}$, it is optimal for bidder 1 to commit to bid $v_{1} / 2$ with probability $v_{1} /\left(2 v_{2}\right)$, and to bid zero with probability $1-v_{1} /\left(2 v_{2}\right)$. Through such an announcement, bidder 1 obtains an expected payoff of $v_{1}^{2} /\left(4 v_{2}\right)$, which is positive and larger than $v_{1}-v_{2}$. Bidder 2's expected payoff is given by $v_{2}-v_{1} / 2$, which is higher than $\max \left\{0, v_{2}-v_{1}\right\}$, her maximum payoff that can be achieved at a Nash equilibrium of the corresponding simultaneous complete-information first-price sealed-bid action. It follows that when $v_{1}<2 v_{2}$, both bidders strictly benefit from this commitment.

It is straightforward to see that bidder 1's maximum expected payoff (resulting from an optimal discrete announcement) is nondecreasing with $t$. The preceding example implies that if $v_{1}<2 v_{2}$, bidder 1 always obtains an expected payoff higher than that achieved in a simultaneous complete-information first-price sealed-bid action, regardless of the value of $t$. As $t$ increases, bidder 1's optimal discrete commitments approaches her optimal commitment, and at the same time, her expected payoff converges to the highest expected payoff characterized in Theorem 1, as shown in the following theorem.

Theorem 4. Let $X^{t}$ be the discrete random variable whose distribution is an optimal solution to (15) with parameter $t$. Bidder 1's expected payoff achieved by committing to $X^{t}$ converges to her highest expected payoff that could be achieved in the unrestricted 
action space $\mathcal{X}$, i.e.,

$$
\lim _{t \rightarrow \infty} \pi_{1}\left(X^{t}, b_{2}^{*}\right)=\sup _{X \in \mathcal{X}} \pi_{1}\left(X, b_{2}^{*}\right)=v_{1}-v_{2}\left(1-e^{-v_{1} / v_{2}}\right),
$$

where the right hand side is the leader's maximum expected payoff characterized in Theorem 1.

\subsection{Multi-bidder case}

Proposition 1 shows that if bidder 1's valuation is less than the second highest valuation, she cannot obtain a positive expected payoff at an SPE, even if she can commit to an arbitrary mixed strategy. The following theorem characterizes bidder 1's optimal announcement for the case where bidder 1's valuation is the highest or the second highest.

Theorem 5. Suppose that bidder 1's valuation is the highest or the second highest, and that bidder 3 has the second highest valuation among the remaining $n-1$ bidders with $v_{3}<v_{2}$. We have the following.

1. Suppose that bidder 2 wins the item if there is a tie. A commitment $\left\{\left(a_{i}^{*}, p_{i}^{*}\right)\right\}_{i=1}^{t}$, which is an optimal solution to the following optimization problem,

$$
\begin{array}{lll}
\text { maximize } & v_{1}\left(1-p_{1}\right)-\sum_{i=1}^{t} a_{i} p_{i} & \\
\text { subject to } & \sum_{i=1}^{t} p_{i}=1, & \\
& p_{i} \geq 0, & i=1, \ldots, t, \\
& a_{1}=0, \quad a_{i}=v_{2}-\frac{\left(v_{2}-v_{3}\right) p_{1}}{\sum_{k=1}^{i} p_{k}}, & i=2, \ldots, t,
\end{array}
$$

together with the strategy profile defined in Eq. (12), form an SPE of the game. Bidder 2 bids $v_{3}$ at the SPE, and obtains an expected payoff of $\left(v_{2}-v_{3}\right) p_{1}^{*}$. 
2. The SPE yields bidder 1 the highest expected payoff among all possible SPEs.

Theorem 6. Suppose that bidder 1's valuation is the highest or the second highest, and that bidder 3 has the second highest valuation among the rest $n-1$ bidders with $v_{3}<v_{2}$. Let $X^{t}$ be the discrete random variable whose distribution is an optimal solution to (17) with parameter $t$. Bidder 1's expected payoff achieved by committing to $X^{t}$ converges to her highest expected payoff, i.e.,

$$
\left.\lim _{t \rightarrow \infty} \pi_{1}\left(X^{t}, \overline{\mathbf{b}}\right)=\sup _{X \in \mathcal{X}} \pi_{1}(X, \overline{\mathbf{b}})=v_{1}-v_{2}\left(1-e^{\left(v_{3}-v_{1}\right) /\left(v_{2}-v_{3}\right)}\right)-v_{3} e^{\left(v_{3}-v_{1}\right) /\left(v_{2}-v_{3}\right)}\right),
$$

where the right hand side is bidder 1's highest expected payoff that could be achieved if she is allowed to make any arbitrary commitment (cf. Theorem 2).

The proof of Theorem 6 parallels that of Theorem 4, and is therefore omitted.

\section{Bayesian Setting with Two Bidders}

In this section, we move away from the full-information model to consider a Bayesian setting where there are two bidders, each agent knows her own valuation, and the leader knows only a prior distribution on the follower's valuation. We assume that the set of possible follower types is a finite set of positive integers, $\{1, \ldots, \Theta\}$. A type- $\theta$ follower has a valuation of $v_{2}^{\theta}$. We arrange the types in ascending order, i.e.,

$$
v_{2}^{1}<v_{2}^{2}<\ldots<v_{2}^{\Theta}
$$

A type- $\theta$ follower's strategy, $b_{2}^{\theta}$, is a mapping from the set of possible commitments, $\mathcal{X}$, to the same set $\mathcal{X}$. The leader (bidder 1 ) chooses a commitment $X_{1}$ so as to maximize her expected payoff $\mathbb{E}\left\{\pi_{1}\left(X_{1}, b_{2}^{\theta}\right)\right\}$, where the expectation is over the realizations of $X_{1}$ and the follower's type. To characterize the SPE that maximizes the leader's expected 
payoff, we consider the following pure strategy of bidder 2 :

$$
b_{2}^{\theta}(X)=\min \left\{\operatorname{argmax}_{b \in\left[0, v_{2}^{\theta}\right]}\left\{F_{X}(b)\left(v_{2}^{\theta}-b\right)\right\}\right\}, \quad \forall X \in \mathcal{X} .
$$

Note that bidder 2 always chooses a best response that is most preferred by the leader. We have shown in Lemma 1 that the strategy defined in (18) is legitimate.

Before presenting the main result of this section, we introduce some notation that will be useful later. Given two nonnegative real numbers $b, s$, and a type $\theta$, we define

$$
g_{b, s}^{\theta}(x) \triangleq \frac{\left(v_{2}^{\theta}-b\right) s}{v_{2}^{\theta}-x}, \quad x \in\left[b, v_{2}^{\theta}(1-s)+b s\right]
$$

Suppose that $s$ is the probability that the leader's bid is no more than $b$. If the CDF of the leader's commitment is as defined in (19), then a type- $\theta$ follower will be indifferent among bids in the interval $\left[b, v_{2}^{\theta}(1-s)+b s\right]$. We note that $g_{b, s}^{\theta}(x)$ is increasing in $x$ and equals 1 at $x=v_{2}^{\theta}(1-s)+b s$. We will show (in the following theorem) that there exists a leader's optimal commitment of the form

$$
F_{\bar{X}_{1}}(x)= \begin{cases}0, & x<0, \\ g_{0, \beta}^{1}(x), & x \in\left[0, a_{2}\right), \\ g_{a_{\theta}, F_{X_{1}}\left(a_{\theta}\right)}^{\Theta}(x), & x \in\left[a_{\theta}, a_{\theta+1}\right), \quad \theta \in\{2, \ldots, \Theta-1\}, \\ g_{a_{\Theta}, F_{X_{1}}\left(a_{\Theta}\right)}^{\Theta}(x), & x \in\left[a_{\Theta}, v_{2}^{\Theta}\left(1-F_{X_{1}}\left(a_{\Theta}\right)\right)\right), \\ 1, & x \geq v_{2}^{\Theta}\left(1-F_{X_{1}}\left(a_{\Theta}\right)\right),\end{cases}
$$

where $\beta$ is the probability mass $\bar{X}_{1}$ assigns to zero, i.e., $\beta=F_{\bar{X}_{1}}(0)$. In Eq. (20), we essentially define a set of functions parameterized by $\Theta$ variables: $\beta$, and $a_{2}, \ldots, a_{\Theta}$. As we will see in the statement of the following theorem, the optimal commitment of 
the form in (20) should make a type- $\theta$ follower bid $a_{\theta}$, and therefore,

$$
F_{\bar{X}_{1}}\left(a_{\theta}\right)\left(v_{2}^{\theta}-a_{\theta}\right)>F_{\bar{X}_{1}}(b)\left(v_{2}^{\theta}-b\right), \quad \forall b \in\left[0, a_{\theta}\right), \quad \theta \in\{2, \ldots, \Theta\} .
$$

We are now ready to characterize the leader optimal commitment (whose CDF satisfies condition (20)) as an optimal solution to the following optimization problem:

$$
\operatorname{maximize} \sum_{\theta=1}^{\Theta} \mathbb{P}(\theta)\left(1-F_{\bar{X}_{1}}\left(a_{\theta}\right)\right)\left(v_{1}-\mathbb{E}\left\{\bar{X}_{1} \mid \bar{X}_{1}>a_{\theta}\right\}\right)
$$

subject to $(21)$,

$$
0 \leq \beta, \quad a_{2} \leq a_{3} \leq \ldots \leq a_{\Theta}
$$

$$
\text { over } \beta, a_{2}, \ldots, a_{\Theta} \text {, }
$$

where $\mathbb{P}(\theta)$ is the probability that the follower is of type $\theta, a_{1}=0$, and $\bar{X}_{1}$ is a random variable whose CDF satisfies condition (20). We will show in the following theorem that under a commitment $\bar{X}_{1}$ that satisfies conditions (20) and (21), a type- $\theta$ follower's best response is to bid $a_{\theta}$, and therefore the objective function in Problem (22) is the leader's expected payoff achieved under commitment $\bar{X}_{1}$.

Theorem 7. We have the following.

1. Suppose that bidder 2 wins the item if there is a tie. The follower's strategy defined in Eq. (18), together with a commitment $\bar{X}_{1}$ whose CDF satisfies condition (20) (with parameters $\beta, a_{2}, \ldots, a_{\Theta}$ an optimal solution to Problem (22)), form an SPE of the game.

2. On the equilibrium path of the SPE, a type-1 follower bids zero, and a type- $\theta$ follower bids $a_{\theta}$, for $\theta=2, \ldots, \Theta$.

3. The above SPE yields bidder 1 the highest expected payoff among all SPEs (of all possible models with different tie-breaking rules).

4. Bidder 1 can approximately secure this highest expected payoff, regardless of the 
tie-breaking rule and the best response chosen by bidder 2. That is, bidder 1's expected payoff achieved at the SPE equals $\bar{\pi}_{1}$ (cf. Definition 1).

The proof of Theorem 7 is deferred to Appendix C. Although the theorem does not provide a closed-form expression of leader optimal commitments, it reduces the leader's decision making problem, which is rather complicated mainly because of her large action space and the lack of information on the follower's valuation, to an optimization problem with $\Theta$ (the number of possible follower's types) variables.

\section{Conclusion}

We consider a variation of the standard single-item sealed-bid first-price auction wherein one bidder publicly commits to a (possibly mixed) strategy before the other bidders act. To our knowledge, we are the first to provide a complete characterization of optimal commitments in any Stackelberg games. We find that the leader's optimal commitment yields both the leader and the bidder with the highest valuation among the rest a utility benefit, when compared with maximum payoff they can obtain in a simultaneous complete-information first-price auction. The characterization we provide gives interesting insight into the effects of commitments on bidder action, bidder utility, and auctioneer revenue. Our results show that even a very restricted form of commitment, which could quite easily be implemented - wherein the leader commits to exit the auction with some probability and to bid a known value with the remaining probability - can yield significant benefits for the top two valued bidders.

Overall, we find that commitment can serve as a powerful coordinating mechanism that allows the top two valued bidders to coordinate, despite the absence of side payments or contracts, with an additional nice property that the net utility benefit of commitment is equally divided between the two bidders. 


\section{A Proofs of Results in Section 3}

\section{A.1 Proof of Lemma 2}

In the subgame that follows a commitment $X_{1}$, let $X_{i}$ denote bidder $i$ 's strategy used in a Nash equilibrium. Suppose that bidder 3 obtains a positive expected payoff at the Nash equilibrium. We will derive a contradiction in the following three steps.

Step 1: Bidder 2 obtains a positive expected payoff at the Nash equilibrium.

At the Nash equilibrium, because bidder 3 obtains a positive expected payoff, we must have

$$
\mathbb{P}\left(\max \left\{X_{1}, X_{4}, \ldots, X_{n}\right\} \leq v_{3}\right)>0 .
$$

Because $X_{3}$ is bidder 3's best response, we have

$$
\mathbb{P}\left(X_{3} \leq v_{3}\right)=1
$$

We therefore conclude that bidder 2 obtains a positive expected payoff at the Nash equilibrium. Actually, given the strategy profile used by the other bidders, bidder 2 is guaranteed to obtain a positive expected payoff by bidding $\left(v_{3}+v_{2}\right) / 2$.

Step 2: The "lower endpoint" of the range of $X_{2}$ is no less than the "lower endpoint" of the range of $X_{3}$.

For a random variable $X \in \mathcal{X}$, we define

$$
F_{X}^{-1}(t)=\min \{x \in[0, \infty): F(x) \geq t\}, \quad t \in[0,1]
$$

Note that because $F_{X}$ is nondecreasing and right-continuous, the set of real numbers 
$\{x: F(x) \geq t\}$ always attains a minimum on $[0, \infty)$. We define the "lower endpoint" of random variable $X_{i}$ 's range as

$$
d_{i} \triangleq \lim _{k \rightarrow \infty} F_{X_{i}}^{-1}(1 / k)
$$

where the limit always exists because the sequence $\left\{F_{X_{i}}^{-1}(1 / k)\right\}$ is nonincreasing and lower bounded by zero. Because bidder 3 obtains a positive expected payoff at the Nash equilibrium, we have $d_{3} \leq v_{3}$.

In this step, we argue that $d_{3} \leq d_{2}$. Suppose not, and we will show that the following strategy is a profitable deviation for bidder 2 :

$$
X_{2}^{\prime}(\omega)= \begin{cases}X_{2}(\omega), & \text { if } \quad X_{2}(\omega)>d_{3} \\ d_{3}+\epsilon, & \text { if } \quad X_{2}(\omega) \leq d_{3}\end{cases}
$$

where $\epsilon$ is a small positive real number. Because $d_{3} \geq d_{2}+\delta$ for some $\delta>0$, there exists some $K>0$ such that $F_{X_{2}}^{-1}(1 / K) \leq d_{3}$, which implies that

$$
F_{X_{2}}\left(d_{3}\right) \geq 1 / K
$$

i.e., the set $\left\{\omega: X_{2}(\omega) \leq d_{3}\right\}$ has a positive measure. It is then straightforward to check that the strategy defined in (24) is a profitable deviation for bidder 2. The strategy profile $\left(X_{2}, \ldots, X_{n}\right)$ is not a Nash equilibrium, and the desired result follows.

Step 3: Bidder 3 cannot obtain a positive expected payoff at the Nash equilibrium.

If $d_{3}<d_{2}$, then there exist some $K>0$ and $\delta>0$ such that $F_{X_{3}}^{-1}(1 / K) \leq d_{2}-\delta$, which implies that

$$
F_{X_{3}}\left(d_{2}-\delta\right) \geq 1 / K
$$


i.e., bidder 3 bids less than $d_{2}$ with positive probability. It follows that there exists some $b_{3} \in\left[0, d_{2}-\delta\right]$ that maximizes bidder 3's expected payoff. The desired result follows from the fact that bidder 3 obtains a zero payoff by submitting a bid that is less than $d_{2}$.

We now consider the case where $d_{3}=d_{2}$. If $F_{X_{2}}$ has an atom at $d_{2}$, i.e., $\mathbb{P}\left(X_{2}=\right.$ $\left.d_{2}\right)>0$, then bidder 2's expected payoff is maximized by bidding $d_{2}$. Because bidder 2 obtains a positive payoff at the Nash equilibrium, it follows that

$$
\mathbb{P}\left(\max \left\{X_{1}, X_{3}, \ldots, X_{n}\right\} \leq d_{2}\right)>0
$$

which implies $\mathbb{P}\left(X_{3}=d_{2}\right)>0$. It is straightforward to check that under the strategy profile $\left(X_{1}, X_{3}, \ldots, X_{n}\right)$ such that

$$
\mathbb{P}\left(\max \left\{X_{1}, X_{3}, \ldots, X_{n}\right\}=d_{2}\right)>0
$$

bidder 2 would like to bid $d_{2}$ with positive probability only when she wins the item if there is a tie. Because bidder 3 bids $d_{2}$ with positive probability, it follows that a pure strategy of bidding $d_{2}$ yields bidder 3 a maximum expected payoff, which is zero.

It remains to consider the case where $d_{3}=d_{2}$ and $F_{X_{2}}$ has no atom at $d_{2}$. If $F_{X_{3}}$ has an atom at $d_{2}$, i.e., $\mathbb{P}\left(X_{3}=d_{2}\right)>0$, then bidder 3 's expected payoff is maximized by bidding $d_{2}$. It follows that bidder 3 achieves a zero payoff at the Nash equilibrium. If $F_{X_{3}}$ has no atom at $d_{2}$, then it is continuous at $d_{2}$. Because $F_{X_{2}}$ is continuous at $d_{2}$ and $F_{X_{2}}\left(d_{2}\right)=0$, for any $\epsilon>0$, there exists some $t>d_{2}$ such that $F_{X_{2}}(t) \leq \epsilon$. It follows from the definition of $d_{2}$ in $(23)$ that $F_{X_{3}}(t)>0$, i.e., bidder 3 's bid lies in the interval $[0, t]$ with positive probability. Therefore, bidder 3's expected payoff achieved at the Nash equilibrium is at most $v_{3} F_{X_{2}}(t) \leq v_{3} \epsilon$. Because the preceding inequality holds for any $\epsilon>0$, we conclude that bidder 3 obtains a zero payoff at the Nash equilibrium. 


\section{A.2 Proof of Lemma 3}

In the subgame that follows a commitment $X_{1}$, let $X_{i}$ denote bidder $i$ 's strategy used in a Nash equilibrium, for $i=2, \ldots, n$. We first consider the case where $\mathbb{P}\left(X_{1}<v_{3}\right)>0$. Lemma 2 shows that bidder 3 obtains a zero payoff at the Nash equilibrium of the subgame. It follows that at the Nash equilibrium, the maximum of the random variables $X_{2}, X_{4}, \ldots, X_{n}$ is larger than or equal to $v_{3}$, with probability 1 . If

$$
\mathbb{P}\left(\max \left\{X_{4}, \ldots, X_{n}\right\} \geq v_{3}\right)<1
$$

then $\mathbb{P}\left(X_{2}\left(X_{1}\right) \geq v_{3}\right)=1$, as desired. For the case where there exists some bidder $i \geq 4$ such that $\mathbb{P}\left(X_{i}\left(X_{1}\right) \geq v_{3}\right)=1$, let $d_{i}$ be the real number defined in (23). Because $\mathbb{P}\left(X_{1}<v_{2}\right)>0$, we must have $d_{i} \leq v_{2}$, otherwise either bidder $i$ or bidder 2 receives a negative expected payoff at the Nash equilibrium (which is impossible). If $d_{i}<v_{2}$, then through an approach similar to that used in Step 2 of the proof of Lemma 2, one can show that $d_{i} \leq d_{2}$, which implies that $\mathbb{P}\left(X_{2}\left(X_{1}\right) \geq v_{3}\right)=1$. If $d_{i}=v_{2}$, then bidder $i$ must never win the item: otherwise she receives a negative expected payoff. It follows that bidder 2 wins the item whenever $X_{1}$ is less than $v_{2} \cdot{ }^{10}$ We therefore have $d_{i}=d_{2}=v_{2}$, i.e., both bidder $i$ and bidder 2 use a pure strategy, $X_{i}\left(X_{1}\right)=X_{2}\left(X_{1}\right)=v_{2}$, and receive a zero expected payoff.

It remains to consider the case with $\mathbb{P}\left(X_{1}<v_{3}\right)=0$. It is easy to check that $d_{1} \geq v_{3}$. Because $\mathbb{P}\left(X_{1}<v_{2}\right)>0$, if bidder 2 receives a positive expected payoff at the Nash equilibrium, then through an approach similar to that used in Step 2 of the proof of Lemma 2, it can be shown that $v_{3} \leq d_{1} \leq d_{2}$, which implies that $\mathbb{P}\left(X_{2}\left(X_{1}\right) \geq v_{3}\right)=1$. If bidder 2 receives a zero payoff at the Nash equilibrium, then there exists some bidder $i \geq 3$ such that $d_{i}=v_{2}$. Because bidder $i$ receives a zero payoff at the Nash equilibrium, it follows that bidder 2 uses a pure strategy $X_{2}\left(X_{1}\right)=v_{2}$.

\footnotetext{
${ }^{10}$ Note that the case $d_{i}=v_{2}$ may occur at a Nash equilibrium only if bidder 2 wins the item at a tie.
} 


\section{A.3 Proof of Proposition 1}

Throughout this paper we assume that bidder 2 has the highest valuation among the $n-1$ bidders (excluding bidder 1 ). Without loss of generality, we let bidder 3 have the second highest valuation. Because $v_{1} \leq v_{3}$, bidder 1 obtains a nonpositive payoff if she commits to bid higher than $v_{3}$, i.e., if she commits to bid a random variable $X$ such that $\mathbb{P}\left(X \geq v_{3}\right)=1$.

Suppose that bidder 1 makes a commitment $X$ such that $\mathbb{P}\left(X \geq v_{3}\right)<1$ at an SPE. Because $\mathbb{P}\left(X<v_{2}\right)>0$, it follows from Lemma 3 that bidder 2 bids at least $v_{3}$ at the SPE. Because $v_{3} \geq v_{1}$, it follows that at the SPE, bidder 1 cannot obtain a positive expected payoff.

\section{A.4 Proof of Theorem 2}

We will prove this theorem in an approach similar to that used for Theorem 1. To argue that $\left(\bar{X}_{1}, \overline{\mathbf{b}}\right)$ is an SPE, we will first show that the strategy profile $\overline{\mathbf{b}}$ is a Nash equilibrium of the subgame that follows any history (the action taken by bidder 1 at the first stage) $X_{1}$. We then show that $\bar{X}_{1}$ maximizes $\pi_{1}\left(X_{1}, \mathbf{b}\right)$ among all possible announcements $X_{1}$ such that $\mathbb{P}\left(X_{1}<v_{2}\right)>0$, through an approach similar to that used in the proof of Theorem 1. Because bidder 1 obtains at most a payoff of $v_{1}-v_{2}$ by committing to some $X_{1}$ such that $\mathbb{P}\left(X_{1}<v_{2}\right)=0$, it follows from the fact that (Part 2 of the theorem)

$$
\pi_{1}\left(\bar{X}_{1}, \mathbf{b}\right)>\max \left\{0, v_{1}-v_{2}\right\}
$$

that $\bar{X}_{1}$ maximizes $\pi_{1}\left(X_{1}, \overline{\mathbf{b}}\right)$ among all $X_{1} \in \mathcal{X}$. This establishes $\left(\bar{X}_{1}, \overline{\mathbf{b}}\right)$ as an SPE.

Step 1: The strategy profile defined in Eq. (12) is a Nash equilibrium of any subgame. 
Given a history $X_{1} \in \mathcal{X}$, we show that the strategy profile $\overline{\mathbf{b}}$ is a Nash equilibrium of the subgame that follows $X_{1}$. For a bidder $i \geq 3$, given bidder 2's strategy $\bar{b}_{2}$, she cannot expect a positive payoff, and therefore $\bar{b}_{i}=v_{i}$ is her best response.

If $\mathbb{P}\left(X_{1}<v_{2}\right)=0$, then bidder 2 cannot expect a positive payoff, and $\bar{b}_{2}(X)=v_{3}$ (which will yield her a zero payoff) is her best response. If $\mathbb{P}\left(X_{1}<v_{2}\right)>0$, it is easy to check the strategy $\bar{b}_{2}$ maximizes bidder 2 's expected payoff, conditioned that bidder 3 bids her own valuation.

Step 2: There exists an optimal commitment under which bidding $v_{3}$ is a best response of bidder 2 .

In Step 2-4, we will show that $\bar{X}$ maximizes $\pi_{1}\left(X_{1}, \overline{\mathbf{b}}\right)$ among all possible announcements $X$ such that $\mathbb{P}\left(X_{1}<v_{2}\right)>0$. Under a commitment $X_{1}$ with $\mathbb{P}\left(X_{1}<v_{2}\right)>0$, Lemma 3 shows that bidder 2 will bid at least $v_{3}$ at an SPE. Suppose that bidder 2 's best response is larger than $v_{3}$, i.e., $\bar{b}_{2}\left(X_{1}\right)>v_{3}$. Given the CDF of the random variable $X_{1}$, we define an alterative random variable $X_{1}^{\prime}$ by

$$
F_{X_{1}^{\prime}}(x)= \begin{cases}0, & \text { if } x<0 \\ F_{X_{1}}\left(\bar{b}_{2}\left(X_{1}\right)\right), & \text { if } 0 \leq x \leq \bar{b}_{2}\left(X_{1}\right), \\ F_{X_{1}}(x), & \text { if } x>\bar{b}_{2}\left(X_{1}\right)\end{cases}
$$

where bidder 1 is committed to bid zero with probability $F_{X_{1}}\left(\bar{b}_{2}\left(X_{1}\right)\right)$ in this alternative announcement. Following Step 1 in the proof of Theorem 1, one can show that bidder 2's best response under the commitment $X_{1}^{\prime}$ is to bid $v_{3}$, and that bidder 1 's expected payoff cannot decrease under the alternative announcement $X_{1}^{\prime}$.

\section{Step 3: Optimality conditions.}


In this step, we show that there exists an optimal commitment, $\bar{X}_{1}$, whose CDF satisfies the following condition:

$$
F_{\bar{X}_{1}}(x)= \begin{cases}0, & \text { if } x<0, \\ F_{\bar{X}_{1}}(0), & \text { if } 0 \leq x \leq v_{3}, \\ \frac{\left(v_{2}-v_{3}\right) F_{\bar{X}_{1}}(0)}{v_{2}-x}, & \text { if } \quad v_{3}<x<v_{2}\left(1-F_{\bar{X}_{1}}(0)\right)+v_{3} F_{\bar{X}_{1}}(0), \\ 1, & \text { if } x \geq v_{2}-\left(v_{2}-v_{3}\right) F_{\bar{X}_{1}}(0) .\end{cases}
$$

We have shown in Step 1 that there exists an optimal commitment under which it is optimal for bidder 2 to bid $v_{3}$. Let $\bar{X}_{1}$ be such an optimal commitment, and we have

$$
\left(v_{2}-v_{3}\right) F_{\bar{X}_{1}}(0) \geq\left(v_{2}-x\right) F_{\bar{X}_{1}}(x), \quad x \geq v_{3},
$$

where the left hand side is bidder 2's expected payoff achieved by bidding $v_{3}$, and the right hand side is her expected payoff achieved by submitting a bid $x \geq v_{3}$. For a random variable $X_{1}$ that satisfies the condition in (26), if the inequality strictly holds for some $x \in\left[v_{3}, v_{2}\left(1-F_{\bar{X}}(0)\right)+v_{3} F_{\bar{X}_{1}}(0)\right]$, i.e., if

$$
F_{X_{1}}(x)<\frac{\left(v_{2}-v_{3}\right) F_{X_{1}}(0)}{v_{2}-x} \leq 1
$$

then it can be shown (through an approach similar to that used in the proof of Theorem 1) $\pi_{1}\left(X_{1}\right) \leq \pi_{1}\left(X_{1}^{\prime}\right)$, where $X_{1}^{\prime}$ is a random variable satisfies conditions (25) with $F_{X_{1}^{\prime}}(0)=F_{X_{1}}(0)$.

Step 4: The probability that bidder 1 should leave the auction equals $e^{\left(v_{3}-v_{1}\right) /\left(v_{2}-v_{3}\right)}$.

For the random variable $\bar{X}_{1}$ defined in (25), we note that the derivative of its CDF, 
$f_{\bar{X}_{1}}(x)$, exists over the domain $\left(v_{3}, v_{2}\left(1-F_{\bar{X}_{1}}(0)\right)+v_{3} F_{\bar{X}_{1}}(0)\right)$ :

$$
f_{\bar{X}_{1}}(x)=\frac{\left(v_{2}-v_{3}\right) \beta}{\left(v_{2}-x\right)^{2}}
$$

where $\beta \triangleq F_{\bar{X}_{1}}(0)$. Through a simple calculation we have

$$
\mathbb{E}\left\{\bar{X}_{1} \mid \bar{X}_{1}>v_{3}\right\}=\frac{1}{1-\beta} \int_{v_{3}}^{v_{2}(1-\beta)+v_{3} \beta} x f_{\bar{X}_{1}}(x) d x=v_{2}+\frac{\left(v_{2}-v_{3}\right) \beta \log \beta}{1-\beta} .
$$

We write bidder 1's expected payoff achieved by committing to bid $\bar{X}_{1}$ as a function of $\beta$ :

$$
\begin{aligned}
\pi_{1}\left(\bar{X}_{1}, \overline{\mathbf{b}}\right) & =\left(v_{1}-\mathbb{E}\left\{\bar{X}_{1} \mid \bar{X}_{1}>v_{3}\right\}\right)(1-\beta) \\
& =\left(v_{1}-v_{2}\right)(1-\beta)-\left(v_{2}-v_{3}\right) \beta \log \beta .
\end{aligned}
$$

The derivative of $\pi_{1}\left(\bar{X}_{1}, \overline{\mathbf{b}}\right)$ with respect to $\beta$ is

$$
v_{3}-v_{1}-\left(v_{2}-v_{3}\right) \log \beta
$$

We therefore conclude that bidder 1's expected payoff is (strictly) maximized at

$$
F_{\bar{X}_{1}}(0)=\beta=e^{\left(v_{3}-v_{1}\right) /\left(v_{2}-v_{3}\right)} .
$$

It follows that the random variable $\bar{X}_{1}$ maximizes bidder 1's expected payoff, $\pi_{1}\left(X_{1}, \overline{\mathbf{b}}\right)$, among all possible announcements $X_{1}$ such that $\mathbb{P}\left(X_{1}<v_{2}\right)>0$. Substituting the value $\beta$ to (28), we have

$$
\pi_{1}\left(\bar{X}_{1}, \overline{\mathbf{b}}\right)=v_{1}-v_{2}\left(1-e^{\left(v_{3}-v_{1}\right) /\left(v_{2}-v_{3}\right)}\right)-v_{3} e^{\left(v_{3}-v_{1}\right) /\left(v_{2}-v_{3}\right)} .
$$

It is straightforward to check that

$$
\pi_{1}\left(\bar{X}_{1}, \overline{\mathbf{b}}\right)>\max \left\{0, v_{1}-v_{2}\right\}
$$


which implies that at $\left(\bar{X}_{1}, \overline{\mathbf{b}}\right)$ bidder 1 obtains an expected payoff that is higher than that achieved in a simultaneous complete-information auction.

Note that bidder 1 obtains at most an expected payoff of $v_{1}-v_{2}$ by committing to a mixed strategy $X$ with $\mathbb{P}\left(X_{1}<v_{2}\right)=0$. It follows from (29) that the random variable $\bar{X}$ maximizes bidder 1's expected payoff, $\pi_{1}\left(X_{1}, \overline{\mathbf{b}}\right)$, among all $X_{1} \in \mathcal{X}$. We conclude that $\left(\bar{X}_{1}, \overline{\mathbf{b}}\right)$ is an SPE.

Lemma 3 shows that at any Nash equilibrium of a simultaneous complete-information sealed-bid first-price auction, the bidder with the highest valuation will bid at least the second highest valuation. Therefore, in the corresponding simultaneous completeinformation first-price auction (where all bidders have the same valuations as in our Stackelberg model), a Nash equilibrium cannot yield bidder 1 an expected payoff higher than $\max \left\{0, v_{1}-v_{2}\right\}$. To prove Part 2 , it suffices to show that

$$
\pi_{2}\left(\bar{X}_{2}, \overline{\mathbf{b}}\right)>\max \left\{0, v_{2}-v_{1}\right\}
$$

It is easy to see that

$$
\pi_{2}\left(\bar{X}_{2}, \overline{\mathbf{b}}\right)=\left(v_{2}-v_{3}\right) \beta=\left(v_{2}-v_{3}\right) e^{\left(v_{3}-v_{1}\right) /\left(v_{2}-v_{3}\right)}
$$

For the case with $v_{1} \geq v_{2}$, Eq. (30) trivially holds. If $v_{1}<v_{2}$, we have

$$
\log \left(\pi_{2}\left(\bar{X}_{2}, \overline{\mathbf{b}}\right)\right)=\log \left(v_{2}-v_{3}\right)+\frac{v_{3}-v_{1}}{v_{2}-v_{3}} \geq \log \left(v_{2}-v_{1}\right)
$$

where the inequality follows from fact that $v_{2}-v_{3}>-\left(v_{3}-v_{1}\right)$, and

$$
\frac{v_{3}-v_{1}}{v_{2}-v_{3}} \leq \log \left(1+\frac{v_{3}-v_{1}}{v_{2}-v_{3}}\right), \quad \frac{v_{3}-v_{1}}{v_{2}-v_{3}} \in(-1,0]
$$

We now turn to prove the Part 3 of the theorem, i.e., the SPE yields bidder 1 the highest expected payoff among all possible SPEs. Let $\left(\hat{X}_{1}, \hat{\mathbf{b}}\right)$ be an SPE. If $\mathbb{P}\left(\hat{X}_{1}<\right.$ 
$\left.v_{2}\right)=0$, then bidder 1's expected payoff is at most $v_{1}-v_{2}$. The desired result,

$$
\pi_{1}\left(\bar{X}_{1}, \overline{\mathbf{b}}\right) \geq \pi_{1}\left(\hat{X}_{1}, \hat{\mathbf{b}}\right)
$$

follows from (29). If $\mathbb{P}\left(\hat{X}_{1}<v_{2}\right)>0$, Lemma 3 shows that $\hat{b}_{2}\left(\hat{X}_{1}\right) \geq v_{3}$ with probability 1. It follows from the definition of $\bar{b}_{2}$ in (13) that

$$
\mathbb{P}\left(\bar{b}_{2}\left(\hat{X}_{1}\right) \leq \hat{b}_{2}\left(\hat{X}_{1}\right)\right)=1
$$

We therefore have

$$
\pi_{1}\left(\bar{X}_{1}, \overline{\mathbf{b}}\right) \geq \pi_{1}\left(\hat{X}_{1}, \overline{\mathbf{b}}\right) \geq \pi_{1}\left(\hat{X}_{1}, \hat{\mathbf{b}}\right)
$$

where the first inequality follows from the definition of an SPE, and the second inequality is true because of (31). Because the preceding argument holds for any arbitrary announcement $\hat{X}_{1}$ and any (possibly mixed) Nash equilibrium $\hat{X}_{2}$ of the subgame that follows $\hat{X}_{1}$, it follows that bidder 1 cannot secure a higher expected payoff, i.e., $\pi_{1}\left(\bar{X}_{1}, \overline{\mathbf{b}}\right) \geq \bar{\pi}_{1}$.

For the last part of the theorem, it remains to show that $\pi_{1}\left(\bar{X}_{1}, \overline{\mathbf{b}}\right) \leq \bar{\pi}_{1}$. We consider a sequence of random variables $\left\{\bar{X}_{1}^{k}\right\}$,

$$
\bar{X}_{1}^{k}(\omega)=\left\{\begin{array}{lll}
\bar{X}_{1}(\omega), & \text { if } \quad \bar{X}_{1}(\omega)=v_{3}, \\
\bar{X}_{1}(\omega)+\frac{1}{k}, & \text { if } \quad \bar{X}_{1}(\omega)>v_{3}
\end{array}\right.
$$

under which bidder 2's unique best response is to bid zero. It is easy to check that

$$
\pi_{1}\left(\bar{X}_{1}, \overline{\mathbf{b}}\right)=\lim _{k \rightarrow \infty} \bar{\pi}_{1}\left(\bar{X}_{1}^{k}, \overline{\mathbf{b}}\right) \leq \bar{\pi}_{1}
$$




\section{B Proof of Results in Sections 4}

\section{B.1 Proof of Theorem 3}

Let $X^{t}$ denote a random variable whose distribution is an optimal solution to (15). To show that $\left(X^{t}, b_{2}^{*}\right)$ is an SPE, we argue that $X^{t}$ maximizes bidder 1's expected payoff, $\pi_{1}\left(X_{1}, b_{2}^{*}\right)$, among all discrete random variables $X_{1}$ in her restricted action space.

Under an announcement $\left\{\left(a_{i}, p_{i}\right)\right\}_{i=1}^{t}$, bidder 2 uses the strategy defined in (1) and submits a bid $a_{j}$ such that

$$
j=\min \left\{\underset{i}{\operatorname{argmax}}\left\{\left(v_{2}-a_{i}\right) \sum_{k=1}^{i} p_{k}\right\}\right\}
$$

Given the announcement $\left\{\left(a_{i}, p_{i}\right)\right\}_{i=1}^{t}$ and bidder 2's best response $a_{j}$, bidder 1's expected payoff is

$$
v_{1}\left(1-\sum_{i=1}^{j} p_{i}\right)-\sum_{i=j+1}^{t} a_{i} p_{i}
$$

We first note that if $a_{m}>v_{1}$ for some $m \leq t$, bidder 1 can obtain a higher payoff by letting $a_{i}=v_{1}$ for $i \geq m$. It follows that bidder 1 should never make an announcement such that $a_{t}>v_{1}$. Given an announcement $\left\{\left(a_{i}, p_{i}\right)\right\}_{i=1}^{t}$ with $a_{1}>0$ and $a_{t} \leq v_{1}$, suppose that $a_{j}>0$ is bidder 2's best response. Consider an alternative announcement

$$
a_{1}^{\prime}=0, \quad p_{1}^{\prime}=\sum_{i=1}^{j} p_{i} ; \quad a_{i}^{\prime}=a_{j+i-1}, \quad p_{i}^{\prime}=p_{j+i-1}, \quad i=2, \ldots, t-j+1 .
$$

Under such an announcement, if bidder 2 bids $a_{i}^{\prime} \geq a_{j}$, she obtains at most the expected payoff that is achieved under the original announcement, $\pi_{2}\left(a_{j}\right)$; on the other hand, she obtains an expected payoff higher than $\pi_{2}\left(a_{j}\right)$ if she bids zero. It follows that under the alternative announcement $\left\{\left(a_{i}^{\prime}, p_{i}^{\prime}\right)\right\}_{i=1}^{t-j+1}$, bidder 2's best response is to bid zero. The alternative announcement, $\left\{\left(a_{i}^{\prime}, p_{i}^{\prime}\right)\right\}_{i=1}^{t-j+1}$, yields bidder 1 an expected payoff higher than that achieved under the original announcement, because the alternative 
announcement reduces bidder 1's expected payment.

We have shown that it is optimal for bidder 1 to choose $a_{1}^{*}=0$, and make bidder 2 's best response equal to zero. It follows that an optimal announcement, $\left\{\left(a_{i}^{*}, p_{i}^{*}\right)\right\}_{i=1}^{t}$, must satisfy

$$
a_{1}^{*}=0, \quad v_{2} p_{1}^{*} \geq\left(v_{2}-a_{i}^{*}\right) \sum_{k=1}^{i} p_{k}^{*}, \quad i=2, \ldots, t .
$$

If one of these inequalities is strict, e.g., if for some $i \geq 2$ we have

$$
v_{2} p_{1}^{*}>\left(v_{2}-a_{i}^{*}\right) \sum_{k=1}^{i} p_{k}^{*}
$$

then $\left\{\left(a_{i}^{*}, p_{i}^{*}\right)\right\}_{i=1}^{t}$ is not optimal: bidder 1 could have obtained a higher expected payoff by choosing some $a_{i}^{\prime}<a_{i}^{*}$ such that

$$
v_{2} p_{1}^{*}=\left(v_{2}-a_{i}^{\prime}\right) \sum_{k=1}^{i} p_{k}^{*}
$$

It follows that

$$
v_{2} p_{1}^{*}=\left(v_{2}-a_{i}^{*}\right) \sum_{k=1}^{i} p_{k}^{*}, \quad i=2, \ldots, t
$$

which implies that

$$
a_{i}=v_{2}-\frac{v_{2} p_{1}}{\sum_{k=1}^{i} p_{k}}, \quad i=2, \ldots, t
$$

That is, bidder 1's optimal announcement satisfies the third constraint in (15).

Through an approach similar to that used in the proof of Part 3 of Theorem 1, it can be shown that the SPE yields bidder 1 the highest expected payoff among all possible SPEs. 


\section{B.2 Proof of Theorem 4}

Let $\bar{X}_{1}: \Omega \rightarrow\left[0, v_{2}\left(1-e^{-v_{1} / v_{2}}\right)\right]$ be the random variable characterized in (2). Theorem 1 shows that

$$
\sup _{X \in \mathcal{X}} \pi_{1}\left(X, b_{2}^{*}\right)=\pi_{1}\left(\bar{X}_{1}, b_{2}^{*}\right)
$$

In Theorem 1 we have shown that $b_{2}^{*}\left(\bar{X}_{1}\right)=0$. Given a positive integer $k$, we define a random variable $Y^{2^{k}+1}: \Omega \rightarrow\left[0, v_{2}\right]$ as follows,

$$
Y^{2^{k}+1}(\omega)= \begin{cases}0 & \text { if } \quad \bar{X}_{1}(\omega)=0, \\ \frac{j+1}{2^{k}} v_{2}, & \text { if } \frac{j}{2^{k}} v_{2}<\bar{X}_{1}(\omega) \leq \frac{j+1}{2^{k}} v_{2}, \quad j \in \mathbb{N} .\end{cases}
$$

For any given $k$, because the range of $Y^{2^{k}+1}$ has a cardinality no more than $2^{k}+$ $1, Y^{2^{k}+1}$ is a discrete random variable. It is straightforward to see that under the announcement $Y^{2^{k}+1}$, bidder 2's best response (under the strategy $b_{2}^{*}$ ) is to bid zero. It follows that bidder 1 wins the item with the same probability, under the two different announcements $\bar{X}$ and $Y^{2^{k}+1}$. Therefore, to argue

$$
\lim _{k \rightarrow \infty} \pi_{1}\left(Y^{2^{k}+1}, b_{2}^{*}\right)=\pi_{1}\left(\bar{X}_{1}, b_{2}^{*}\right)
$$

it suffices to show that

$$
\lim _{k \rightarrow \infty} \mathbb{E}\left\{Y^{2^{k}+1}\right\}=\mathbb{E}\left\{\bar{X}_{1}\right\}
$$

It is easy to check that the sequence $\left\{Y^{2^{k}+1}\right\}$ is nonincreasing in $k$, and converges to $\bar{X}$ pointwise. We let $Z_{k}=Y^{2^{1}+1}-Y_{2^{k}+1}$. The sequence $\left\{Z_{k}\right\}$ is nonnegative, nondecreasing, and converges to $Y^{2^{1}+1}-\bar{X}$ pointwise. According to monotone convergence theorem [22], we have

$$
\lim _{k \rightarrow \infty} \mathbb{E}\left\{Y^{2^{1}+1}-Y^{2^{k}+1}\right\}=\lim _{k \rightarrow \infty} \mathbb{E}\left\{Z_{k}\right\}=\mathbb{E}\left\{Y^{2^{1}+1}-\bar{X}\right\}
$$


Because $Y^{2^{1}+1} \in\left[0, v_{2}\right], \mathbb{E}\left\{Y^{2^{1}+1}\right\}$ is a nonnegative constant. Therefore, the preceding relation implies the result in (35). The desired result follows from (34) and the fact that

$$
\pi_{1}\left(X^{2^{k}+1}, b_{2}^{*}\right) \geq \pi_{1}\left(Y^{2^{k}+1}, b_{2}^{*}\right), \quad k=1,2 \ldots
$$

\section{B.3 Proof of Theorem 5}

We have shown in the proof of Theorem 2 that the strategy profile $\overline{\mathbf{b}}$ is a Nash equilibrium of the subgame that follows any history $X_{1}$. Let $X^{t}$ denote the discrete random variable characterized in (17). To argue that $\left(X^{t}, \overline{\mathbf{b}}\right)$ is an SPE, we will show that $X^{t}$ maximizes bidder 1's expected payoff, $\pi_{1}\left(X_{1}, \overline{\mathbf{b}}\right)$, among all possible commits $X_{1}$ in her restricted action space.

Suppose first that bidder 1 commits to a random variable $X_{1}=\left\{\left(a_{i}, p_{i}\right)\right\}_{i=1}^{t}$. It is then straightforward to check that bidder 2's best response is among the set $\left\{v_{3}, a_{1}, \ldots, a_{t}\right\}$. We let $j$ denote the largest index such that $a_{j} \leq \bar{b}_{2}\left(X_{1}\right)$, i.e.,

$$
j=\max _{i}\left\{i \in\{1, \ldots, t\}: a_{i} \leq \bar{b}_{2}\left(X_{1}\right)\right\}
$$

Bidder 1's expected payoff is given by

$$
v_{1}\left(1-\sum_{i=1}^{j} p_{i}\right)-\sum_{i=j+1}^{t} a_{i} p_{i}
$$

In Theorem 3 we have shown that bidder 1 should never make an announcement such that $a_{t}>v_{1}$. We therefore assume that $a_{t} \leq v_{1}$. If $a_{1}>0$ or $\bar{b}_{2}\left(X_{1}\right)>v_{3}$, we consider the following commitment

$$
a_{1}^{\prime}=0, \quad p_{1}^{\prime}=\sum_{i=1}^{j} p_{i}, \quad a_{i}^{\prime}=a_{j+i-1}, \quad p_{i}^{\prime}=p_{j+i-1}, \quad i=2, \ldots, t-j+1,
$$

where $j$ is the positive integer defined in (36). Under this alternative commitment, it is 
straightforward to see that bidder 2's best response is $v_{3}$ (according to the strategy $\bar{b}_{2}$ ). It follows that this alternative commitment yields bidder 1 a higher expected payoff than $X_{1}$. We have shown that it is optimal for bidder 1 to choose $a_{1}^{*}=0$, and make bidder 2's best response equal to $v_{3}$.

Because bidder 2's best response is $v_{3}$ under an optimal announcement $\left\{\left(a_{i}^{*}, p_{i}^{*}\right)\right\}_{i=1}^{t}$, we have

$$
a_{1}^{*}=0 ; \quad\left(v_{2}-v_{3}\right) p_{1}^{*} \geq\left(v_{2}-a_{i}^{*}\right) \sum_{k=1}^{i} p_{k}^{*}, \quad i=2, \ldots, t .
$$

If one of these inequalities is strict, e.g.,

$$
\left(v_{2}-v_{3}\right) p_{1}^{*}>\left(v_{2}-a_{i}^{*}\right) \sum_{k=1}^{i} p_{k}^{*}
$$

then $\left\{\left(a_{i}^{*}, p_{i}^{*}\right)\right\}_{i=1}^{t}$ is not optimal: bidder 1 could obtain a higher expected by choosing some $a_{i}^{\prime}<a_{i}^{*}$ such that

$$
\left(v_{2}-v_{3}\right) p_{1}^{*}=\left(v_{2}-a_{i}^{\prime}\right) \sum_{k=1}^{i} p_{k}^{*}
$$

It follows that

$$
\left(v_{2}-v_{3}\right) p_{1}^{*}=\left(v_{2}-a_{i}^{*}\right) \sum_{k=1}^{i} p_{k}^{*}, \quad i=2, \ldots, t,
$$

which implies the third constraint in (17). Through an approach similar to that used in the proof of Part 3 of Theorem 2, it can be shown that the SPE yields bidder 1 the highest expected payoff among all possible SPEs.

\section{Proof of Theorem 7}

We first show that a higher valued follower always bids no less than a lower valued follower, in the following lemma. This result will be useful in our proof.

Lemma 4. For two types $\theta<\theta^{\prime}$, we have $b_{2}^{\theta}(X) \leq b_{2}^{\theta^{\prime}}(X)$, under every commitment $X \in \mathcal{X}$ 
Proof. Under a commitment $X$, let $b_{2}^{\theta}(X)$ denote the best response of a type- $\theta$ follower defined in (18). It follows that bidder 2 obtains strictly lower expected payoff if she bids less than $b_{2}^{\theta}(X)$, i.e.,

$$
F_{X}\left(b_{2}^{\theta}(X)\right)\left(v_{2}^{\theta}-b_{2}^{\theta}(X)\right)>F_{X}(b)\left(v_{2}^{\theta}-b\right), \quad \forall b \in\left[0, b_{2}^{\theta}(X)\right)
$$

Because

$$
F_{X}(b)\left(v_{2}^{\theta^{\prime}}-b\right)-F_{X}(b)\left(v_{2}^{\theta}-b\right)=F_{X}(b)\left(v_{2}^{\theta^{\prime}}-v_{2}^{\theta}\right),
$$

is nonnegative and non-decreasing in $b$, for $b \in\left[0, b_{2}^{\theta}(X)\right)$ we have

$$
F_{X}\left(b_{2}^{\theta}(X)\right)\left(v_{2}^{\theta^{\prime}}-b_{2}^{\theta}(X)\right)-F_{X}\left(b_{2}^{\theta}(X)\right)\left(v_{2}^{\theta}-b_{2}^{\theta}(X)\right) \geq F_{X}(b)\left(v_{2}^{\theta^{\prime}}-b\right)-F_{X}(b)\left(v_{2}^{\theta}-b\right) .
$$

It then follows from (38) that

$$
F_{X}\left(b_{2}^{\theta}(X)\right)\left(v_{2}^{\theta^{\prime}}-b_{2}^{\theta}(X)\right)>F_{X}(b)\left(v_{2}^{\theta^{\prime}}-b\right), \quad \forall b \in\left[0, b_{2}^{\theta}(X)\right),
$$

which implies that $b_{2}^{\theta^{\prime}}(X) \geq b_{2}^{\theta}(X)$.

We now proceed to prove Theorem 7 . We first show Parts 1 and 2 by constructing a leader's optimal commitment that satisfies condition (20). Within this proof, we will frequently use the fact that under a commitment $X$, a type- $\theta$ follower's expected payoff (received by bidding $b$ ) is $F_{X}(b)\left(v_{2}^{\theta}-b\right)$. We say a commitment $X$ is more "aggressive" than another commitment $X^{\prime}$, if

$$
F_{X}(x) \leq F_{X^{\prime}}(x), \quad x \in[0, \infty)
$$

A more aggressive commitment always yields the leader a higher expected payment. Fixing the bid submitted by the follower, it is easy to see that a more aggressive commitment yields the leader higher winning probability, and at the same time, decreases 
the follower's expected payoff.

It is straightforward to check that there exists a leader's optimal commitment such that $F_{X_{1}}\left(v_{1}\right)=1$, under which the leader bids more than its valuation with zero probability. Under such a commitment $X$, it follows from Lemma 4 that $b_{2}^{1}(X) \leq$ $b_{2}^{2}(X) \leq \ldots b_{2}^{\Theta}(X) \leq v_{1}$. We define a modified commitment $X^{r}$ whose CDF is

$$
F_{X^{r}}(x)=g_{0, F_{X}\left(b_{2}^{1}(X)\right)}^{1}(x)=\frac{v_{2}^{1} F_{X}\left(b_{2}^{1}(X)\right)}{v_{2}^{1}-x}, \quad x \in\left[0, v_{2}^{1}\left(1-F_{X}\left(b_{2}^{1}(X)\right)\right)\right],
$$

which, by definition (cf. the definition in (19)), makes a type-1 follower indifferent from bidding any real number in the interval $\left[0, v_{2}^{1}\left(1-F_{X}\left(b_{2}^{1}(X)\right)\right)\right]$. The CDF of $X^{r}$ is illustrated in Fig. 4 (the red curve).

For any $x \in[0, \infty)$, because $b_{2}^{1}(X)$ is a best response, we have

$$
F_{X}(x) \leq \frac{\left(v_{2}^{1}-b_{2}^{1}(X)\right) F_{X}\left(b_{2}^{1}(X)\right)}{v_{2}^{1}-x} \leq \frac{v_{2}^{1} F_{X}\left(b_{2}^{1}(X)\right)}{v_{2}^{1}-x}=F_{X^{r}}(x)
$$

Because $X^{r}$ is less aggressive than the original commitment $X$, there exists some $\tilde{b} \leq$ $b_{2}^{2}(X)$ such that

$$
F_{X^{r}}(\tilde{b})=F_{X}\left(b_{2}^{2}(X)\right)
$$

Based on the modified commitment $X^{r}$, we define a new commitment with the following CDF:

$$
F_{X^{b}}(x)= \begin{cases}F_{X^{r}}(x), & 0 \leq x \leq \tilde{b}, \\ F_{X}\left(b_{2}^{2}(X)\right), & \tilde{b}<x \leq b_{2}^{2}(X), \\ F_{X}(x), & b_{2}^{2}(X)<x,\end{cases}
$$

An illustration of the CDF defined above is given in Fig. 5. It is easy to check that $X^{b}$ is less aggressive than the original commitment $X$. Indeed, the commitment $X^{b}$ yields the leader at least the same expected payoff as $X$, because $X^{b}$ is less aggressive than 


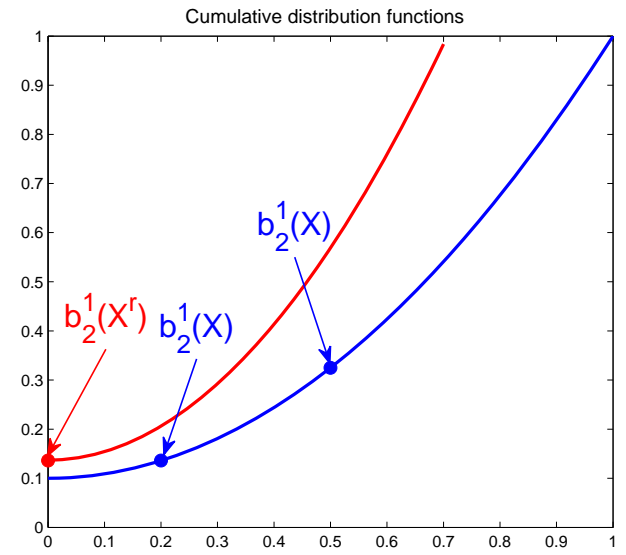

Figure 4: The CDF of the original commitment $X$ (in blue), and a modified CDF $F_{X^{r}}=$ $g_{0, F_{X}\left(b_{2}^{1}(X)\right)}^{1}$ (in red).

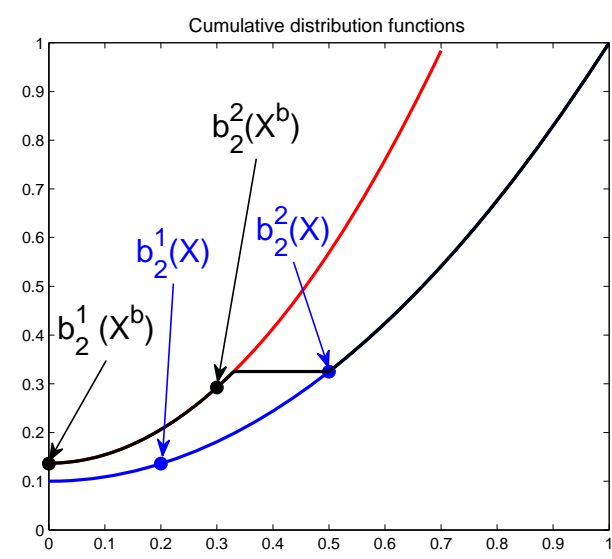

Figure 5: The CDF of the original commitment $X$ (in blue), and a modified CDF $F_{X^{r}}=g_{0, F_{X}\left(b_{2}^{1}(X)\right)}^{1}$ (in red). The black curve illustrates the CDF of another modified commitment $X^{b}$ (defined in (39)).

$X$, and yields the leader higher winning probability, i.e., $F_{X^{b}}\left(b_{2}^{\theta}\left(X^{b}\right)\right) \leq F_{X}\left(b_{2}^{\theta}(X)\right)$, for every $\theta$.

We now prove the fact that $F_{X^{b}}\left(b_{2}^{\theta}\left(X^{b}\right)\right) \leq F_{X}\left(b_{2}^{\theta}(X)\right)$, for every $\theta$. For any $\theta \geq 2$, we have $b_{2}^{\theta}\left(X^{b}\right) \leq b_{2}^{\theta}(X)$, because

$$
F_{X^{b}}(x)=F_{X}(x), \quad x \in\left(b_{2}^{2}(X), \infty\right) ; \quad F_{X^{b}}(x) \geq F_{X}(x), \quad x \in\left[0, b_{2}^{2}(X)\right],
$$

which implies that a type- $\theta$ follower achieves the same expected payoff (under both $X$ and $\left.X^{b}\right)$ if she bids larger than $b_{2}^{2}(X)$, but could potentially obtain a higher expected payoff by biding less than $b_{2}^{2}(X)$ under commitment $X^{b}$. We also note that under commitment $X^{b}$, the follower (of any type) would not like to bid in the interval $\left(\tilde{b}, b_{2}^{2}(X)\right]$. These two facts establish $F_{X^{b}}\left(b_{2}^{\theta}\left(X^{b}\right)\right) \leq F_{X}\left(b_{2}^{\theta}(X)\right)$, for every $\theta \geq 2$. Because $b_{2}^{1}\left(X^{b}\right) \leq b_{2}^{2}\left(X^{b}\right) \leq b_{2}^{2}(X)$, it follows from the construction of $X^{b}$ that $b_{2}^{1}\left(X^{b}\right)=0$, which implies that $F_{X^{b}}\left(b_{2}^{1}\left(X^{b}\right)\right)=F_{X}\left(b_{2}^{1}(X)\right)$.

Taking $X^{b}$ as an "original" commitment, an optimal commitment that satisfies 
condition (20) can be constructed by repeating the above process. The next first step is to define a new commitment $X^{r 2}$ whose CDF agrees with that of $X^{b}$ over the interval $\left[0, b_{2}^{2}\left(X^{b}\right)\right]$, and makes a type-2 follower indifferent over $\left[b_{2}^{2}\left(X^{b}\right), v_{2}^{2}\left(1-F_{X^{b}}\left(b_{2}^{2}\left(X^{b}\right)\right)\right)\right)$, i.e.,

$$
F_{X^{r 2}}=g_{b_{2}^{2}\left(X^{b}\right), F_{X^{b}}\left(b_{2}^{2}\left(X^{b}\right)\right)}^{2}(x), \quad v_{2} \in\left[b_{2}^{2}\left(X^{b}\right), v_{2}^{2}\left(1-F_{X^{b}}\left(b_{2}^{2}\left(X^{b}\right)\right)\right)\right) .
$$

It is straightforward to check that under the new commitment $X^{r 2}$ is less aggressive than $X^{b}$, and

$$
b_{2}^{1}\left(X^{r 2}\right)=b_{2}^{1}\left(X^{b}\right)=0, \quad b_{2}^{2}\left(X^{r 2}\right)=b_{2}^{2}\left(X^{b}\right) .
$$

If $\Theta=2$, then we are done: $X^{r 2}$ satisfies condition (20) (with parameter $a_{2}$ equal to $\left.b_{2}^{2}\left(X^{b}\right)\right)$ and yields the leader at least the same expected payoff as the original commitment $X$. Otherwise, the next step is to construct a commitment $X^{b 2}$ as follows

$$
F_{X^{b 2}}(x)= \begin{cases}F_{X^{r 2}}(x), & 0 \leq x \leq \hat{b}, \\ F_{X^{b}}\left(b_{2}^{3}\left(X^{b}\right)\right), & \hat{b}<x \leq b_{2}^{3}\left(X^{b}\right), \\ F_{X^{b}}(x), & b_{2}^{3}\left(X^{b}\right)<x\end{cases}
$$

where $\hat{b}$ is a real number no more than $b_{2}^{3}(X)$ such that $F_{X^{r 2}}(\hat{b})=F_{X^{b}}\left(b_{2}^{3}\left(X^{b}\right)\right)$. We repeat this process and can finally reach a commitment $X^{r \Theta}$, which satisfies condition (20) and is at least as good as the original commitment $X$. We note from the construction process that under the optimal commitment $X^{r \Theta}$ that satisfies condition (20), the constraints in (21) suffice that

$$
b_{2}^{\theta}\left(X^{r \Theta}\right)=a_{\theta}, \quad \theta=2, \ldots, \Theta
$$

where $\left\{a_{\theta}\right\}_{\theta=2}^{\ominus}$ are parameters of condition (20). We have established the first two parts of the theorem. The proof of Parts 3 and 4 is similar to that for Parts 3 and 4 of 
Theorem 1, and is therefore omitted.

\section{References}

[1] L. H. Baldwin, R. C. Marshall, and J. F. Richard, Bidder collusion at forest service timber sales. Journal of Political Economy, 105(1997), 657-699.

[2] M. Pesendorfer, A study of collusion in first-price auctions. Review of Economic Studies, 67(2000), 381-411.

[3] R. C. Marshall and L. M. Marx, Bidder collusion. Journal of Economic Theory, 133(2007), 374-402.

[4] P. Eso and J. Schummer, Bribing and signalling in second price auctions. Games and Economic Behavior, 47(2004), 299-324.

[5] G. Kivetz and Y. Tauman, Simple collusive agreements in one-shot first-price auctions. Games and Economic Behavior, 9(2010), 138-149.

[6] R. P. McAfee and J. McMillan, Bidding rings. American Economic Review, 82(1992), 579-599.

[7] M. Aoyagi, Bid rotation and collusion in repeated auctions. Journal of Economic Theory, 112(2003), 79-105.

[8] A. Skrzypacz and H. Hopenhayn, Tacit collusion in repeated auctions. Journal of Economic Theory, 114(2004), 153169.

[9] S. Rachmilevitch, Endogenous bid rotation in repeated auctions. Journal of Economic Theory, 148(2013), 1714-1725.

[10] H. von Stackelberg, Marktform und Gleichgewicht. Berlin, Germany: SpringerVerlag, 1934.

[11] T. Schelling, The Strategy of Conflict. Cambridge, MA, USA: Harvard University Press, 1960. 
[12] B. von Stengel and S. Zamir, Leadership games with convex strategy sets. Games and Economic Behavior, 69(2010), 446-457.

[13] A. Rubinstein and A. Wolinsky, Decentralized trading, strategic behaviour and the Walrasian outcome. Review of Economic Studies, 57(1990), 63-78.

[14] D. R. Vincent, Modelling competitive behavior. Rand Journal of Economics, 23(1992), 590-599.

[15] M. Tennenholtz, Program equilibrium. Games and Economic Behavior, 49(2004), 363-373.

[16] D. Fudenberg and D. K. Levine, Reputation and equilibrium selection in games with a patient player. Econometrica, 57(1989), 759-778.

[17] K. M. Schmidt, Reputation and equilibrium characterization in repeated games with conflicting interests. Econometrica, 61(1993), 325-351.

[18] R. Evans and J. P. Thomas, Reputation and experimentation in repeated games with two long-run players. Econometrica, 65(1997), 1153-1173.

[19] M. Maschler, A price leadership method for solving the inspector's nonconstantsum game. Naval Research Logistics Quarterly, 13(1966), 11-33.

[20] T. Basar and G. J. Olsder, Dynamic Noncooperative Game Theory. London: Academic Press, 1982.

[21] M. J. Osborne and A. Rubinstein, A Course in Game Theory. Cambridge, MA, USA: MIT Press, 1994.

[22] D. P. Bertsekas and J. N. Tsitsiklis, Introduction to Probability (2nd. ed.). Belmont, MA, USA: Athena Scientific, 2008. 